\title{
OPERATOR PENCILS ON THE ALGEBRA OF DENSITIES
}

\author{
A. BIGGS AND H. M. KHUDAVERDIAN
}

\begin{abstract}
In this paper we continue to study equivariant pencil liftings and differential operators on the algebra of densities. We emphasize the role that the geometry of the extended manifold plays where the algebra of densities is a special class of functions. Firstly we consider basic examples. We give a projective line of diff $(M)$-equivariant pencil liftings for first order operators, and the canonical second order self-adjoint lifting. Secondly we study pencil liftings equivariant with respect to volume preserving transformations. This helps to understand the role of self-adjointness for the canonical pencils. Then we introduce the Duval-Lecomte-Ovsienko (DLO)-pencil lifting which is derived from the full symbol calculus of projective quantisation. We use the DLO-pencil lifting to describe all regular proj-equivariant pencil liftings. In particular the comparison of these pencils with the canonical pencil for second order operators leads to objects related to the Schwarzian.
\end{abstract}

\section{INTRODUCTION}

We consider pencils of operators on the algebra of densities passing through a given operator.

Firstly let us fix notations. Let $\mathcal{F}_{\lambda}(M)$ be the space of densities of weight $\lambda$ on a manifold $M$ for $\lambda$ an arbitrary real number. If $s=s(x)|D x|^{\lambda} \in \mathcal{F}(M)$ then under changing of local coordinates $x=x\left(x^{\prime}\right)$,

$$
\boldsymbol{s}=s(x)|D x|^{\lambda}=s\left(x\left(x^{\prime}\right)\right)\left|\operatorname{det}\left(\frac{\partial x}{\partial x^{\prime}}\right)\right|^{\lambda}\left|D x^{\prime}\right|^{\lambda} .
$$

The space of functions on $M$ is $\mathcal{F}_{0}(M)$, densities of weight $\lambda=0$. We can multiply densities of different weights. If $\boldsymbol{s}_{1}=s_{1}(x)|D x|^{\lambda_{1}}$ and $\boldsymbol{s}_{2}=s_{2}(x)|D x|^{\lambda_{2}}$ are densities of weights $\lambda_{1}, \lambda_{2}$ respectively then $\boldsymbol{s}=\boldsymbol{s}_{1} \cdot \boldsymbol{s}_{2}=s_{1}(x) s_{2}(x)|D x|^{\lambda_{1}+\lambda_{2}}$ is a density of weight $\lambda_{1}+\lambda_{2}$. We come to the algebra $\mathcal{F}(M)=\oplus_{\lambda} \mathcal{F}_{\lambda}(M)$ of densities of all weights on the manifold $M$.

We also consider differential operators on the whole algebra of densities. The operators of order zero are operators of multiplication by an arbitrary non-zero density; $L$ is an operator of order $\leqslant n+1$ on $\mathcal{F}(M)$ if for an arbitrary density $\boldsymbol{s}$, the commutator $[L, \boldsymbol{s}]=L \circ \boldsymbol{s}-\boldsymbol{s} \circ L$ is an operator of order $\leqslant n$. For example consider the linear operator $\widehat{w}$ on $\mathcal{F}(M)$ defined by

$$
\widehat{w}(s)=\lambda s \quad \text { if } s \in \mathcal{F}_{\lambda}(M), \quad \widehat{w} \text { is the weight operator } .
$$

It is easy to see that $\widehat{w}$ is a first order operator on the algebra of densities, since $\widehat{w}\left(\boldsymbol{s}_{1} \cdot \boldsymbol{s}_{2}\right)=$ $\boldsymbol{s}_{1} \widehat{w}\left(\boldsymbol{s}_{2}\right)+\boldsymbol{s}_{2} \widehat{w}\left(\boldsymbol{s}_{1}\right)$. For example the operator $\widehat{\Delta}=\left(\widehat{w}^{2}+1\right) \frac{\partial^{2}}{\partial x^{2}}+\frac{\partial}{\partial x}$ is a fourth order operator

2000 Mathematics Subject Classification. 15A15, 58A50, 81R99.

Key words and phrases. differential operator, algebra of densities, pencil of operators, self-adjoint operators, equivariant maps on operators. 
on the algebra of densities on the line $\mathbb{R}$. Its restriction to the space of densities of weight $\lambda$ is the operator

$$
\Delta_{\lambda}=\left.\widehat{\Delta}\right|_{\widehat{w}=\lambda}=\left(\lambda^{2}+1\right) \frac{d^{2}}{d x^{2}}+\frac{d}{d x},
$$

which is an operator of order 2 on the space $\mathcal{F}_{\lambda}(\mathbb{R})$. We denote by $\mathcal{D}^{(n)}(M)$ the space of linear differential operators of order $\leqslant n$ on the space $\mathcal{F}_{\lambda}(M)$ of densities of weight $\lambda$, and we denote by $\mathcal{D}^{(n)}(\widehat{M})$ the space of operators of order $\leqslant n$ on the algebra $\mathcal{F}(M)$ (the latter notation will become clear soon).

One can consider a pencil of operators (operator pencil) $\left\{\Delta_{\lambda}\right\}$, i.e. a family of operators depending on the parameter $\lambda$, such that for each $\lambda, \Delta_{\lambda}$ is a differential operator on $\mathcal{F}_{\lambda}$. An operator $\widehat{\Delta}$ on the algebra of densities $\mathcal{F}(M)$ defines the pencil of operators:

$$
\widehat{\Delta} \mapsto\left\{\Delta_{\lambda}\right\}: \Delta_{\lambda}=\left.\widehat{\Delta}\right|_{\widehat{w}=\lambda},
$$

such that dependence on $\lambda$ is polynomial. We consider only pencils depending polynomially on $\lambda$ and we identify operators $\widehat{\Delta}$ on the algebra of densities with their corresponding pencils. (For details see [2] and [8].)

It is useful to consider the fibre bundle $\widehat{M} \rightarrow M$ which is the frame bundle of the determinant bundle over the manifold $M$. One can identify the algebra of densities $\mathcal{F}(M)$ with the algebra of quasipolynomials on the extended manifold $\widehat{M}$ (see [6]). In local coordinates use a formal variable $t$ instead $|D x|$. An arbitrary density $s \in \mathcal{F}(M)$ can be identified with function $\sum s_{r}(x) t^{\lambda_{r}}$ on the extended manifold $\widehat{M}$, which is quasipolynomial on fibre variable $t$. We use the local coordinates $\left(x^{i}, t\right)$ on $\widehat{M}$. Local coordinates in the bundle change in the following way:

$$
\left(x^{i^{\prime}}, t^{\prime}\right): \quad x^{i^{\prime}}=x^{i^{\prime}}\left(x^{i}\right), \quad t^{\prime}=t^{\prime}\left(x^{i}, t\right)=\operatorname{det}\left(\frac{\partial x^{i^{\prime}}}{\partial x^{i}}\right) t .
$$

Differential operators on the algebra of densities can be identified with differential operators on quasipolynomials. In particular the weight operator, (11), has the form $\widehat{w}=t \frac{\partial}{\partial t}$.

Differential operators on algebra of densities have natural grading by their weights. We say that an operator $\widehat{\Delta}$ has weight $\delta$ if it maps the spaces $\mathcal{F}_{\lambda}(M)$ to the spaces $\mathcal{F}_{\lambda+\delta}(M)$. (An operator $\widehat{\Delta}$ has weight $\delta$ iff $[\widehat{w}, \widehat{\Delta}]=\widehat{w} \circ \widehat{\Delta}-\widehat{\Delta} \circ \widehat{w}=\delta \widehat{\Delta}$.)

It is also important to distinguish vertical operators. An operator $\widehat{\Delta}$ is a vertical operator if it commutes with multiplication by an arbitrary function $f: \widehat{\Delta}(f \boldsymbol{s})=f \widehat{\Delta}(\boldsymbol{s})$. A differential operator $\widehat{\Delta}$ of weight $\delta$ has the local appearance $\widehat{\Delta}=t^{\delta} \widehat{\Delta}^{\prime}\left(x^{k}, \partial_{i}, \widehat{w}\right)$. We will focus on operators of weight $\delta=0$. Vertical operator of weight $\delta=0$ have the global appearance $\widehat{\Delta}=\sum \widehat{w}^{i} c_{i}(x)$, where the $c_{i}(x)$ are scalar functions.

The algebra $\mathcal{F}(M)$ of densities is endowed with a canonical scalar product.

If $\boldsymbol{s}_{1}=s_{1}(x)|D x|^{\lambda_{1}}$ and $\boldsymbol{s}_{2}=s_{2}(x)|D x|^{\lambda_{2}}$ are two densities with compact support then

$$
\left\langle\boldsymbol{s}_{1}, \boldsymbol{s}_{2}\right\rangle=\left\{\begin{array}{l}
\int_{M} s_{1}(x) s_{2}(x)|D x|, \quad \text { if } \quad \lambda_{1}+\lambda_{2}=1, \\
0 \quad \text { if } \quad \lambda_{1}+\lambda_{2} \neq 1 .
\end{array}\right.
$$

This construction turns out to be a very important tool when studying the geometry of differential operators on $M$ (see [6], [7].) In particular the scalar product defines the adjoint 
of linear operators on the algebra of densities. A linear operator $\widehat{\Delta}$ acting on densities has an adjoint $\widehat{\Delta}^{*}:\left\langle\widehat{\Delta} s_{1}, s_{2}\right\rangle=\left\langle\boldsymbol{s}_{1}, \widehat{\Delta}^{*} \boldsymbol{s}_{2}\right\rangle$. It is evident that $\left(x^{k}\right)^{*}=x^{k}$ and $\partial_{i}^{*}=-\partial_{i}$. One can see that for the weight operator $\widehat{w}, \widehat{w}^{*}=1-\widehat{w}$. Indeed let $s_{1}$ and $s_{2}$ be two arbitrary densities of weights $\lambda_{1}$ and $\lambda_{2}$ respectively. Then $\left\langle\widehat{w} \boldsymbol{s}_{1}, \boldsymbol{s}_{2}\right\rangle=\lambda_{1}\left\langle\boldsymbol{s}_{1}, \boldsymbol{s}_{2}\right\rangle$, and $\left\langle\boldsymbol{s}_{1}, \widehat{w}^{*} \boldsymbol{s}_{2}\right\rangle=$ $\left\langle\boldsymbol{s}_{1},(1-\widehat{w}) \boldsymbol{s}_{2}\right\rangle=\left(1-\lambda_{2}\right)\left\langle\boldsymbol{s}_{1}, \boldsymbol{s}_{2}\right\rangle$, and $\lambda_{1}\left\langle\boldsymbol{s}_{1}, \boldsymbol{s}_{2}\right\rangle=\left(1-\lambda_{2}\right)\left\langle\boldsymbol{s}_{1}, \boldsymbol{s}_{2}\right\rangle$. (If $\lambda_{1}+\lambda_{2} \neq 1$ then $\left\langle\boldsymbol{s}_{1}, \boldsymbol{s}_{2}\right\rangle=0$, if $\lambda_{1}+\lambda_{2}=1$ then $\lambda_{1}=1-\lambda_{2}$.) 1

Our task is to construct a map $\widehat{\Pi}$ which maps each operator on densities of a given weight $\lambda_{0}$ to a pencil of operators which passes through this operator:

$$
\widehat{\Pi}: \quad \mathcal{D}_{\lambda_{0}}^{(n)} \ni \Delta \mapsto \widehat{\Delta}=\widehat{\Pi}(\Delta)=\left\{\Delta_{\lambda}\right\}, \text { such that }\left.\widehat{\Pi}(\Delta)\right|_{\widehat{w}=\lambda_{0}}=\Delta .
$$

We call such a map $\widehat{\Pi}$ a pencil lifting map.

We say that a pencil lifting map $\widehat{\Pi}$ is regular on the space of operators of order $\leqslant n$ if this map takes values in operators of order $\leqslant n$ :

$$
\widehat{\Pi}: \quad \mathcal{D}_{\lambda_{0}}^{(n)}(M) \rightarrow \mathcal{D}^{(n)}(\widehat{M}) .
$$

To make the problem well-defined we put restrictions on the pencil lifting map. We consider regular pencil lifting maps which are equivariant with respect to some Lie subalgebra of vector fields [2]. Let us briefly recall what this means.

A pencil lifting map is equivariant with respect to a subgroup $G$ of the group $\operatorname{Diff}(M)$ of diffeomorphisms of $M$, i.e. it is a $G$-lifting, if for every diffeomorphism $\varphi \in G, \widehat{\Pi}\left(\Delta^{\varphi}\right)=$ $(\widehat{\Pi}(\Delta))^{\varphi}$. If $\varphi$ is a diffeomorphism which is infinitesimally closed to the identity, $\varphi=1+\varepsilon \mathbf{K}$, where $\mathbf{K}$ is a vector field on $M$, then invariance with respect to diffeomorphism $\varphi$ implies that

$$
\operatorname{ad}_{\mathbf{K}}(\widehat{\Pi}(\Delta))=\widehat{\Pi}\left(\operatorname{ad}_{\mathbf{K}}(\Delta)\right)
$$

where operation $\operatorname{ad}_{\mathbf{K}}$ is defined by condition that for every density $\boldsymbol{s}, \operatorname{ad}_{\mathbf{K}}(\Delta) \boldsymbol{s}=\mathcal{L}_{\mathbf{K}}(\Delta \boldsymbol{s})-$ $\Delta\left(\mathcal{L}_{\mathbf{K}} s\right) . \quad\left(\mathcal{L}_{\mathbf{K}}\right.$ is the Lie derivative with respect to vector field $\mathbf{K}$.) We say that a pencil lifting map $\widehat{\Pi}$ is equivariant with respect to Lie algebra $\mathcal{G} \subseteq \operatorname{diff}(M)$, a $\mathcal{G}$ - lifting, if the condition (5) holds for every vector field $\mathbf{K} \in \mathcal{G}$.

Remark 1. We consider regular pencil lifting maps which are equivariant with respect to Lie algebras. Often the results will be valid for corresponding groups.

Here we consider pencil lifting maps which are equivariant with respect to

- the algebra diff $(M)$ of all vector fields on $M$,

- the algebra sdiff $(M)=\operatorname{sdiff}_{\rho}(M)$ of all divergenceless vector fields on $M$ with respect to some volume form $\boldsymbol{\rho}$,

- the algebra $\operatorname{proj}(M)$ of projective vector fields on a projective manifold $M$.

Recall that a vector field $\mathbf{X}$ is divergenceless with respect to the volume form $\boldsymbol{\rho},\left(\boldsymbol{\rho} \in \mathcal{F}_{1}(M)\right)$ if the volume form $\boldsymbol{\rho}$ is invariant with respect to the vector field $\mathbf{X}, \mathcal{L}_{\mathbf{X}} \boldsymbol{\rho}=0$. The divergence of a vector field with respect to $\boldsymbol{\rho}$ is defined by the formula

$$
\operatorname{div}{ }_{\boldsymbol{\rho}} \mathbf{X}=\frac{\mathcal{L}_{\mathbf{X} \boldsymbol{\rho}}}{\boldsymbol{\rho}}=\partial_{i} X^{i}(x)+X^{i}(x) \partial_{i} \log \rho(x) .
$$

\footnotetext{
${ }^{1}$ Note that bilinear form (3) is not positive definite. E.g. for density $s=f(x) \frac{|D x|^{\lambda}-|D x|^{1-\lambda}}{\sqrt{2}},\left(\lambda \neq \frac{1}{2}\right)$ we have that $\langle s, s\rangle=-\int_{M} f^{2}(x) D x$.
} 
The Lie algebra sdiff $\rho(M)$ is a Lie subalgebra of all vector fields of $M$ such that they preserve the volume form $\rho$ on $M$.

A projective manifold of dimension $d$ which is locally a projective space $\mathbb{R} P^{d}$. In local projective coordinates $x^{i}=\left(x^{1}, \ldots, x^{d}\right)$ the Lie algebra $\operatorname{proj}(M)$ is generated by the vector fields

$$
\partial_{i}, \quad x^{i} \partial_{k}, \quad x^{i} x^{k} \partial_{k}, \quad(i, k=1, \ldots, d) .
$$

(Two different local projective coordinates in a vicinity of a point are related by equations $\left.x^{i^{\prime}}=\frac{a_{j}^{i} x^{j}+b^{i}}{c_{k} x^{k}+d}, i, i^{\prime}=1, \ldots . d.\right)$

In what follows we consider regular pencil liftings equivariant with respect to these algebras. We also focus our attention on the self-adjointness of pencil lifting maps.

It should be noted that regular pencil lifting maps can sometimes be described by a full symbol map. Let us explain what this means in more detail.

Let $\mathcal{G}$ be an arbitrary Lie subalgebra of vector fields on $M$. Consider (if it exists) a $\mathcal{G}$ equivariant full symbol map $\sigma_{\lambda}^{(n)}$ from the space $\mathcal{D}_{\lambda}^{(n)}(M)$ of differential operators (of weight $\delta=0$ ) on densities of weight $\lambda$ to the symbol space of contravariant symmetric tensor fields of rank $\leqslant n$ on $M$. The symbol space can be identified with the space of functions on $T^{*} M$ which are polynomials of order $\leqslant n$ in the fibre variables (momenta). The full symbol map $\sigma_{\lambda}^{(n)}$ is a $\mathcal{G}$-equivariant map that prolongs the principal symbol map:

$$
\text { for arbitrary } \mathbf{K} \in \mathcal{G}, \quad \sigma^{(n)}\left(\operatorname{ad}_{\mathbf{K}} \Delta\right)=\mathcal{L}_{\mathbf{K}} \sigma_{\lambda}^{(n)}(\Delta),
$$

and

$$
\text { for arbitrary } \Delta \in \mathcal{D}_{\lambda}^{(k)}(M) \subseteq \mathcal{D}_{\lambda}^{(n)}(M)(k \leqslant n), \sigma_{\lambda}^{(n)}(\Delta)=\Sigma_{\text {princ. }}^{(k)}(\Delta)+\ldots,
$$

where the ellipsis means tensors of rank $<k$, and $\Sigma_{\text {princ. }}^{(k)}$ is the principal symbol: for arbitrary $\Delta=S^{i_{1} \ldots i_{k}} \partial_{i_{1}} \ldots \partial_{i_{k}}+\ldots$,

$$
\Sigma_{\text {princ. }}^{(k)}(\Delta)=S^{i_{1} \ldots i_{k}} \xi_{i_{1}} \ldots \xi_{i_{k}} .
$$

The inverse of such a full symbol map defines a $\mathcal{G}$-equivariant quantisation map $Q_{\lambda}^{(n)}=$ $\left(\sigma_{\lambda}^{(n)}\right)^{-1}$ on the space of symbols. (See the analysis in section 5 for details).

It is well-known that there is no $\operatorname{diff}(M)$-equivariant full symbol map for $n \geqslant 2$. On the other hand there exists a full symbol map $\sigma_{\lambda}^{(n)}$ for arbitrary $n$ and $\lambda$ in the case if $\mathcal{G}$ is the algebra of projective vector fields, or the algebra of conformal vector fields on $\mathbb{R}^{d}$, and this full symbol map is uniquely defined by its equivariance with respect to this algebra. These are famous results on equivariant quantisation due to Duval, Lecomte and Ovsienko (see [3] and [10] and also the book [12]).

The existence of $\mathcal{G}$-equivariant full symbol map $\sigma_{\lambda}^{(n)}$ for all $\lambda$, determines the corresponding pencil lifting map on the spaces $\mathcal{D}_{\lambda}^{(n)}(M)$ of operators on densities of weight $\lambda$ :

$$
\widehat{\Pi}_{\lambda}: \forall \Delta \in \mathcal{D}_{\lambda}^{(n)}(M),\left.\quad\left(\widehat{\Pi}_{\lambda}(\Delta)\right)\right|_{\widehat{w}=\mu}=Q_{\mu}^{(n)} \circ \sigma_{\lambda}^{(n)}(\Delta), \quad Q_{\mu}^{(n)}=\left(\sigma_{\mu}^{(n)}\right)^{-1} .
$$

This pencil lifting can be defined for arbitrary $n$. It is evidently equivariant with respect to the algebra $\mathcal{G}$. One can see that this is regular pencil lifting. We call this pencil lifting the Duval-Lecomte-Ovsienko (DLO)-pencil lifting (see in more details in the section 5).

The DLO-pencil lifting can be factored through a symbol map. Of course not every pencil lifting can be factored through symbol map. Recall that regular pencil $\widehat{\Pi}$ lifting defined on 
$\mathcal{D}_{\lambda}^{(n)}(M)$ is strictly regular (see [2]) if its restriction on any subspace $\mathcal{D}_{\lambda}^{(k)}(M) \subseteq \mathcal{D}_{\lambda}^{(n)}(M)$ is also regular:

$$
\widehat{\Pi}: \quad \forall k \leqslant n, \mathcal{D}_{\lambda}^{(k)}(M) \rightarrow \mathcal{D}_{\lambda}^{(k)}(\widehat{M})
$$

One can see that DLO-pencil pencil lifting is not only regular but also strictly regular pencil lifting on all the spaces $\mathcal{D}_{\lambda}^{(n)}(M)$.

One can see that a regular pencil lifting which can be factored through a symbol map is strictly regular. Being strictly regular is a necessary but not a sufficient condition for a pencil lifting to be factored. However this simple criterion allows us to observe the fact that a pencil lifting cannot be factored through the full symbol map.

The paper is set out in the following way:

In the next two sections we consider regular diff $(M)$-pencil liftings of operators of order $n=1,2$. Firstly we consider regular pencil liftings for first order operators and come to a projective line of diff $(M)$-equivariant liftings. Next we study the canonical pencil for second order operators. Here we construct this canonical pencil as a Laplace-Beltrami like-operator on extended manifold $\widehat{M}$ and study its geometry using a Kaluza-Klein like formalism.

Higher order regular diff $(M)$-pencil liftings $(n \geqslant 3)$ are impossible in general (see [9], [11]). In the fourth section we describe the family of all regular pencil liftings equivariant with respect to volume preserving transformations. Unlike diff $(M)$-pencil liftings these pencil liftings are defined for operators of all orders. Within this family is a distinguished lifting which can be used to study the role of self-adjointness for canonical pencils.

In the fifth section using the projective full symbol map we study the Duval-LecomteOvsienko (DLO)-pencil lifting, and on the base of it we construct all regular self-adjoint and anti-self-adjoint proj-equivariant pencil liftings. In particular we analyze a family of self-adjoint pencil liftings for second order operators, and comparing it with the canonical self-adjoint pencil lifting come to geometrical objects whose transformation law is governed by a multidimensional Schwarzian.

Throughout this paper by default we consider operators of weight zero. Some of these results can be generalised for non-zero weight.

This paper is based on the talk of one of the authors (HMK) at the conference "Algebraic topology and abelian functions" in honor of Professor V.M. Buchstaber in June 2013. Many results of the next three sections of this article were already discussed in our paper [2], and here we consider them under a different light. Results of the last section are new.

\section{Acknowledgment}

We are grateful to organisers of the conference "Algebraic topology and abelian functions" for inviting us to this conference and encouraging us to write this text. We are grateful also to V.Ovsienko and T.Voronov for useful discussions.

\section{PENCIL Lifting FOR FIRST ORDER OPERATORS}

We begin by describing the geometry of first order operators on algebra $\mathcal{F}(M)$. (See also [6], 8].)

Let $\widehat{L}$ be a first order operator on the algebra of densities of weight $\delta$. Then $\widehat{L}=\widehat{\mathbf{X}}+F$, where $F=\widehat{L}(1)$ is a density of weight $\delta$ and $\widehat{\mathbf{X}}$ a vector field of weight $\delta$ on the extended manifold $\widehat{M} ; \widehat{\mathbf{X}}\left(\boldsymbol{s}_{1} \cdot \boldsymbol{s}_{2}\right)=\widehat{\mathbf{X}}\left(\boldsymbol{s}_{1}\right) \cdot \boldsymbol{s}_{2}+\boldsymbol{s}_{1} \widehat{\mathbf{X}}\left(\boldsymbol{s}_{2}\right)$. In components $\widehat{\mathbf{X}}=t^{\delta}\left(X^{i}(x) \partial_{i}+X^{0}(x) \widehat{w}\right)$. 
Using the canonical scalar product, (3), one can assign to the vector field $\widehat{\mathbf{X}}$, its adjoint, the first order operator

$$
\widehat{\mathbf{X}}^{*}=\left[t^{\delta}\left(X^{i}(x) \partial_{i}+X^{0}(x) \widehat{w}\right)\right]^{*}=t^{\delta}\left(-X^{i}(x) \partial_{i}-\widehat{w} X^{0}-\partial_{i} X^{i}(x)+(1-\delta) X^{0}(x)\right) .
$$

Thus we come to the canonical divergence of a vector field $\widehat{\mathbf{X}}$ on $\widehat{M}$ :

$$
\operatorname{div} \widehat{\mathbf{X}}=-\left(\widehat{\mathbf{X}}+\widehat{\mathbf{X}}^{*}\right)=t^{\delta}\left(\partial_{i} X^{i}(x)+(\delta-1) X^{0}(x)\right) .
$$

In the case that $\widehat{\mathbf{X}}=\widehat{\mathbf{X}}(x, t)=X^{i}(x, t) \partial_{i}+X^{0}(x, t) \widehat{w}$ is an arbitrary vector field (weight is not fixed) then this formula reads:

$$
\operatorname{div} \widehat{\mathbf{X}}=-\left(\widehat{\mathbf{X}}+\widehat{\mathbf{X}}^{*}\right)=\partial_{i} X^{i}(x, t)+(\widehat{w}-1) X^{0}(x, t) .
$$

Notice that $\widehat{\mathbf{X}}$ is divergenceless, $\operatorname{div} \widehat{\mathbf{X}}=0$, iff $\widehat{\mathbf{X}}$ is anti-self-adjoint.

Now let us focus our attention on the case when $\delta=0$. In this case the vector field $\widehat{\mathbf{X}}$ projects to a vector field $\mathbf{X}$ on the manifold $M: \mathbf{X}(f):=\widehat{\mathbf{X}}(f)$ for every function $f$ on $M$. Similarly an arbitrary vector field $\mathbf{X}$ on $M$ can be uniquely lifted to a vector field $\widehat{\mathbf{X}}$ on $\widehat{M}$ such that $\widehat{\mathbf{X}}$ projects to $\mathbf{X}$ and $\widehat{\mathbf{X}}^{*}=-\widehat{\mathbf{X}}^{*} \Leftrightarrow \operatorname{div} \widehat{\mathbf{X}}=0$. We denote such a lift of $\mathbf{X}$ by $\widehat{\mathcal{L}}_{\mathbf{X}}$ :

$$
X^{i} \partial_{i}=\mathbf{X} \mapsto \widehat{\mathcal{L}}_{\mathbf{X}}=X^{i}(x) \partial_{i}+\widehat{w} \partial_{i} X^{i}(x), \quad\left(\operatorname{div} \widehat{\mathcal{L}}_{\mathbf{X}}=0\right) .
$$

$\widehat{\mathcal{L}}_{\mathbf{X}}$ is the Lie derivative along the vector field $\mathbf{X}$ : for every density $s=s(x)|D x|^{\lambda}$,

$$
\left.\widehat{\mathcal{L}}_{\mathbf{X}}(s)=\left(X^{i}(x) \partial_{i}+\widehat{w} \partial_{i} X^{i}(x)\right) s(x)|D x|^{\lambda}=\left(X^{i}(x) \partial_{i} s(x)+\lambda \partial_{i} X^{i}(x) s(x)\right)\right)|D x|^{\lambda} .
$$

Thus we see that every vector field $\widehat{\mathbf{X}}$ on $\widehat{M}$ of weight $\delta=0$ is a sum of a Lie derivative $\widehat{\mathcal{L}}_{\mathbf{X}}$ and a vertical vector field

$$
\widehat{\mathbf{X}}=X^{i} \partial_{i}+\widehat{w} X^{0}=\widehat{\mathcal{L}}_{\mathbf{X}}+\widehat{w}\left(X^{0}(x)-\partial_{i} X^{i}(x)\right) .
$$

The function $X^{0}(x)-\partial_{i} X^{i}(x)$ is a scalar function, it does not change under coordinate transformations. Respectively every operator $\widehat{L}$ of order $\leqslant 1$ and of weight $\delta=0$ can be decomposed as

$$
\widehat{L}=\widehat{\mathcal{L}}_{\mathbf{X}}+\widehat{w} S_{1}+S_{2},
$$

where $S_{1}$ and $S_{2}$ are scalar functions; $\mathbf{X}$ is a vector field on $M$, projection of vector field $\widehat{\mathbf{X}}$, $\widehat{L}=\widehat{\mathbf{X}}+S_{2}$, and $\widehat{\mathbf{X}}=\widehat{\mathcal{L}}_{\mathbf{X}}+\widehat{w} S_{1}$ due to (10). This decomposition leads to the construction of canonical pencil lifting for the space of first order operators.

Namely pick an arbitrary $\lambda$ and consider the space $\mathcal{D}_{\lambda}^{(1)}$ of first order operators acting on densities of weight $\lambda$ (as usual we consider only operators of weight $\delta=0$ ), and let $L$ be an arbitrary operator in this space. It defines the vector field $\mathbf{X}$ on $M$ such that $\mathbf{X}(f) \boldsymbol{s}=L(f \boldsymbol{s})-f L(\boldsymbol{s})$, where $\boldsymbol{s}$ is an arbitary density of weight $\lambda$. In local coordinates $L=X^{i}(x) \partial_{i}+F(x)$ Consider the Lie derivative corresponding to the vector field $\mathbf{X}, \mathcal{L}_{\mathbf{X}}^{\lambda}=$ $\left.\widehat{\mathcal{L}}_{\mathbf{X}}\right|_{\widehat{w}=\lambda}$. The difference $L-\mathcal{L}_{\mathbf{X}}=S$ is a zeroth order operator, a scalar function $S$. Hence we arrive at the decomposition of the linear operator $L \in \mathcal{D}_{\lambda}^{(1)}(M)$ into a sum of a Lie derivative and a scalar function:

$$
L=X^{i} \partial_{i}+F(x)=\underbrace{\left(X^{i}(x) \partial_{i}+\lambda \partial_{i} X^{i}(x)\right)}_{\text {Lie derivative } \mathcal{L}_{\mathbf{X}}^{\lambda}}+\underbrace{\left(F(x)-\lambda \partial_{i} X^{i}(x)\right)}_{\text {scalar function } S(x)} .
$$


(We have supposed that $L$ is an operator of weight $\delta=0$.)

Comparing this decomposition with the decomposition (11) we come to the following canonical pencil lifting map defined on the spaces $\mathcal{D}_{\lambda}^{(1)}$ : for every $L=X^{i}(x) \partial_{i}+F(x)$,

$$
\widehat{\Pi}_{\lambda}(L)=\widehat{\mathcal{L}}_{\mathbf{X}}+\left(F(x)-\lambda \partial_{i} X^{i}(x)\right) .
$$

Using this lifting we can define a family of diff $(M)$-equivariant liftings. For every linear operator $L \in \mathcal{D}_{\lambda}^{(1)}, L=X^{i}(x) \partial_{i}+F(x)$, (weight $\delta=0$ ) we define

$$
\widehat{\Pi}_{\lambda}^{[p: q]}(L)=\underbrace{\left(X^{i}(x) \partial_{i}+\widehat{w} \partial_{i} X^{i}(x)\right)}_{\widehat{\mathcal{L}}_{\mathbf{X}}}+\frac{p \widehat{w}+q}{p \lambda+q} \underbrace{\left(F(x)-\lambda \partial_{i} X^{i}(x)\right)}_{S(x)}, \quad\left(\frac{q}{p} \neq-\lambda\right),
$$

where $[p: q]$ is a point on the projective line $\mathbb{R} P^{1}$.

It is evident that for all pencil liftings $\left\{\widehat{\Pi}_{\lambda}^{[p: q]}\right\}$ the condition (41) is obeyed, and all these pencil liftings are evidently diff $(M)$-equivariant since they are defined via canonical constructions. This construction describes all regular diff $(M)$-pencil of first order operators.

The pencil lifting maps $\left\{\widehat{\Pi}_{\lambda}^{[p: q]}\right\}$ can be considered as a map on the space of parameters

$$
(\underbrace{\mathbb{R} P^{1}}_{[p: q]} \times \underbrace{\mathbb{R}}_{\text {weights }}) \backslash \underbrace{\mathbb{R}}_{\frac{p}{q} \neq-\lambda}=\mathbb{R}^{2} .
$$

This family possesses the following distinguished liftings:

i) the pencil lifting $\widehat{\Pi}^{[p: q]}$ for $[p: q]=[0: 1]$ given above (13),

$$
\widehat{\Pi}_{\lambda}^{[0: 1]}(L)=\widehat{\Pi}\left(X^{i}(x) \partial_{i}+F(x)\right)=\widehat{\mathcal{L}}_{\mathbf{X}}+S(x),
$$

ii) the anti-self-conjugate pencil lifting $\widehat{\Pi}^{[p: q]}$ for $[p: q]=[2:-1]$,

$$
\widehat{\Pi}_{[p: q]}^{\lambda}(L)=\widehat{\Pi}\left(X^{i}(x) \partial_{i}+F(x)\right)=\widehat{\mathcal{L}}_{\mathbf{X}}+\frac{2 \widehat{w}-1}{2 \lambda-1} S(x), \quad \lambda \neq \frac{1}{2}, \quad \widehat{\Pi}^{*}=-\widehat{\Pi} .
$$

iii) the affine line of pencil liftings

$$
\widehat{\Pi}_{\lambda}^{c}(L)=\widehat{\Pi}_{\lambda}^{[p: q]}(L)=\Pi\left(X^{i}(x) \partial_{i}+F(x)\right)=\widehat{\mathcal{L}}_{\mathbf{X}}+S(x)+c(\widehat{w}-\lambda) S(x),
$$

where $c$ is a point on affine line:

$$
[p: q]=\left[1: \frac{1}{c}-\lambda\right], \text { in particular }[p: q]=[0: 1] \text { if } c=0 .
$$

Remark 2. The diff ( $M$ )-equivariant pencil lifting (13) is the DLO-pencil lifting (77) for first order operators. Respectively pencil liftings (14) are a special case of regular proj-invariant pencil liftings. (See in detail section 5.)

Remark 3. One can attempt similar analysis for operators of arbitrary weight (for detail see [1]) 


\section{Pencil Liftings of SECOND ORder operators}

We describe the geometry of second order operators on the algebra of densities $\mathcal{F}(M)$.

An arbitrary differential operator on the algebra of densities is a sum of a self-adjoint and anti-self-adjoint operator:

$$
\widehat{\Delta}=\underbrace{\frac{\widehat{\Delta}+\widehat{\Delta}^{*}}{2}}_{\text {self-adjoint }}+\underbrace{\frac{\widehat{\Delta}-\widehat{\Delta}^{*}}{2}}_{\text {anti-self-adjoint }} .
$$

Let $\widehat{\Delta}$ be an arbitrary second order operator on the algebra of densities, $\mathcal{F}(M), \widehat{\Delta} \in \mathcal{D}^{(2)}(M)$ and $\widehat{\Delta} \notin \mathcal{D}^{(1)}(M)$. Then one can see that it is a sum of a second order self-adjoint operator and first order anti-self-adjoint operator. First order anti-self-adjoint operators, which are related with Lie derivatives, were analyzed in the previous section. We now focus on second order self-adjoint operators. Let

$$
\widehat{\Delta}=\underbrace{S^{i k}(x) \partial_{i} \partial_{k}+2 B^{i}(x) \partial_{i} \widehat{w}+C(x) \widehat{w}^{2}}_{\text {second order derivatives }}+\underbrace{D^{i}(x) \partial_{i}+E(x) \widehat{w}}_{\text {first order derivatives }}+F(x), \quad\left(\widehat{w}=t \partial_{t}\right) .
$$

be a second order operator on the algebra of densities. As usual we shall consider the case when the operator $\widehat{\Delta}$ has weight $\delta=0$. The principal symbol of this operator is

$$
\widehat{S}(x, t)=\left(\begin{array}{ll}
S^{i k}(x) & B^{i}(x) t \\
B^{k}(x) t & C(x) t^{2}
\end{array}\right) .
$$

We assign to the principal symbol $\widehat{S}$ the following operator on the algebra of densities:

$$
\widehat{\Delta}_{\widehat{S}}: \quad \widehat{\Delta}_{\widehat{S}}(\boldsymbol{s})=\operatorname{div}(\widehat{S} d \boldsymbol{s}),
$$

where $\boldsymbol{s}=\boldsymbol{s}(x, t)$ is an arbitrary density which is identified with a quasi-polynomial on the extended manifold $\widehat{M}, d s$ is the differential of this density, it is a 1-form (covector) on the bundle space $\widehat{M} ; \widehat{S} d \boldsymbol{s}$ is the vector field on $\widehat{M}$ corresponding to the covector $d \boldsymbol{s}$ raised by $\widehat{S}$, and div is the canonical divergence (9). In local coordinates

$$
\begin{gathered}
d \boldsymbol{s}(x, t)=\partial_{i} \boldsymbol{s}(x, t) d x^{i}+\partial_{t} \boldsymbol{s}(x, t) d t, \\
\widehat{S}(d \boldsymbol{s})=\left(S^{i k}(x) \partial_{k} \boldsymbol{s}(x, t)+B^{i}(x) t \partial_{t} \boldsymbol{s}(x, t)\right) \partial_{i}+\left(B^{k}(x) t \partial_{k} \boldsymbol{s}(x, t)+C(x) t^{2} \partial_{t} \boldsymbol{s}(x, t)\right) \partial_{t}= \\
=\left(S^{i k}(x) \partial_{k} \boldsymbol{s}(x, t)+B^{i}(x) \widehat{w} \boldsymbol{s}(x, t)\right) \partial_{i}+\left(B^{k}(x) \partial_{k} \boldsymbol{s}(x, t)+C(x) \widehat{w} \boldsymbol{s}(x, t)\right) \widehat{w}, \\
\text { and } \widehat{\Delta}_{\widehat{S}}(\boldsymbol{s})=\operatorname{div}(\widehat{S} d \boldsymbol{s})= \\
\partial_{i}\left(S^{i k}(x) \boldsymbol{s}(x, t)+\widehat{w} B^{i}(x) \boldsymbol{s}(x, t)\right)+(\widehat{w}-1)\left(B^{i}(x) \partial_{i} \boldsymbol{s}(x, t)+C(x)(\widehat{w} \boldsymbol{s}(x, t))\right)= \\
S^{i k}(x) \partial_{i} \partial_{k}+\partial_{k} S^{k i}(x) \partial_{i}+(2 \widehat{w}-1) B^{i}(x) \partial_{i}+\widehat{w} \partial_{k} B^{k}(x)+\widehat{w}(\widehat{w}-1) C(x) .
\end{gathered}
$$

It is important to note that the canonical operator $\widehat{\Delta}_{\widehat{S}}$ is self-adjoint. This can be checked directly. It is evident that this operator obeys the normalisation condition $\widehat{\Delta}_{\widehat{S}}(1)=0$. Suppose now that the operator $\widehat{\Delta}$ in (15) is also self-adjoint, $\widehat{\Delta}^{*}=\widehat{\Delta}$. Then the operator $\widehat{\Delta}-\widehat{\Delta}_{\widehat{S}}$ is self-adjoint. Both these operators have the same principal symbol. Hence the self-adjoint operator $\widehat{\Delta}-\widehat{\Delta}_{\widehat{S}}$ is of order $\leqslant 1$. On the other hand an operator of order 1 
cannot be self-adjoint. This implies that $\widehat{\Delta}-\widehat{\Delta}_{\widehat{S}}$ is an operator of multiplication by a scalar function. Thus $\widehat{\Delta}-\widehat{\Delta}_{\widehat{S}}=\widehat{\Delta}(1)=F(x)$. We come to the following

Proposition 1. The canonical operator $\widehat{\Delta}=\widehat{\Delta}_{\widehat{S}}$ on the algebra of densities defined by equation (16) is self-adjoint and obeys the normalisation condition

$$
\widehat{\Delta}(1)=0 \text {. }
$$

An arbitrary second order self-adjoint operator $\widehat{\Delta}, \widehat{\Delta}^{*}=\widehat{\Delta}$ which obeys normalisation condition (18) is uniquely defined by its principal symbol $\widehat{S}, \widehat{\Delta}=\widehat{\Delta}_{\widehat{S}}$.

Remark 4. Canonical divergence (9) can be considered as the divergence with respect to 'generalised volume form' induced by the scalar product (3) (see for details [7]). One can say that formula (16) defines the canonical self-adjoint operator as the Laplace-Beltrami operator on the extended manifold $\widehat{M}$ corresponding to an 'upper Riemannian metric' $\widehat{S}$ and the canonical 'generalised volume form'.

We now analyze the geometrical meaning of the components of the principal symbol $\widehat{S}$. Under changing of local coordinates $x^{\prime}=x^{\prime}(x), t^{\prime}=t^{\prime}(x, t)=\operatorname{det}\left(\frac{\partial x^{i^{\prime}}(x)}{\partial x^{i}}\right) t$ (see equations (2) )

$$
\partial_{i}=\frac{\partial x^{i^{\prime}}(x)}{\partial x^{i}} \partial_{i^{\prime}}+\partial_{i} \log J \widehat{w}, \quad \widehat{w}=t \partial_{t}=t^{\prime} \partial_{t^{\prime}}, \quad \text { where } J=\operatorname{det}\left(\frac{\partial x^{i^{\prime}}}{\partial x^{i}}\right) .
$$

Hence components of the principal symbol (a symmetric contravariant tensor on the extended manifold) transform as follows:

$$
\begin{gathered}
S^{i^{\prime} k^{\prime}}\left(x^{\prime}\right)=\frac{\partial x^{i^{\prime}}(x)}{\partial x^{i}} \frac{\partial x^{k^{\prime}}(x)}{\partial x^{k}} S^{i k}\left(x\left(x^{\prime}\right)\right), \\
B^{i^{\prime}}\left(x^{\prime}\right)=\frac{\partial x^{i^{\prime}}(x)}{\partial x^{i}}\left(B^{i}\left(x\left(x^{\prime}\right)\right)+S^{i k}\left(x\left(x^{\prime}\right)\right) \partial_{k} \log J\right), \\
C^{\prime}\left(x^{\prime}\right)=C\left(x\left(x^{\prime}\right)\right)+2 B^{i}\left(x\left(x^{\prime}\right)\right) \partial_{i} \log J+\partial_{i} \log J S^{i k}\left(x\left(x^{\prime}\right)\right) \partial_{k} \log J .
\end{gathered}
$$

$S^{i k}(x)$ is a contravariant symmetric tensor field on the base manifold $M$. The operator $\widehat{\Delta}$ defines a pencil of second order operators $\left\{\Delta_{\lambda}\right\}, \Delta_{\lambda}=S^{i k} \partial_{i} \partial_{k}+\ldots$, and all these operators on $M$ have the same principal symbol $S^{i k}$. (We suppose that the tensor field $S^{i k}(x)$ does not vanish. If $S^{i k} \equiv 0$, then $\widehat{\Delta}=(2 \widehat{w}-1) \widehat{\mathcal{L}}_{B}-\widehat{w}(\widehat{w}-1)\left(C(x)-2 \partial_{k} B^{k}(x)\right)$.

To study the geometrical meaning of the components $B^{i}(x)$ and $C(x)$ of the operator (16) it is useful to consider a connection on the fibre bundle $\widehat{M} \rightarrow M$. Let $\nabla$ be an arbitrary connection. It defines a covariant derivative of densities with respect to vector fields. If $s$ is a density of weight $\lambda$, and $\mathbf{X}=X^{i}(x) \partial_{i}$ a vector field on $M$, then

$$
\nabla_{\mathbf{X}}(\boldsymbol{s})=\left(X^{i}(x) \partial_{i} s(x)+\lambda \gamma_{i}(x) X^{i}(x) s(x)\right)|D x|^{\lambda} .
$$

For an arbitrary density $\boldsymbol{s}=\boldsymbol{s}(x, t)$ (identified with a quasipolynomial on $\widehat{M}$ )

$$
\nabla_{\mathbf{X}} \boldsymbol{s}(x, t)=\left(X^{i}(x) \partial_{i} \boldsymbol{s}(x, t)+\widehat{w} \gamma_{i}(x) X^{i}(x) \boldsymbol{s}(x, t)\right) .
$$

The components $\gamma_{i}(x)$ of the connection are defined by the equation $\gamma_{i}(x)|D x|=\nabla_{\partial_{i}}|D x|$. The connection $\nabla$ defines a lifting of vector fields from $M$ to horizontal vector fields on $\widehat{M}$. 
The corresponding connection form, $\Omega$ on $\widehat{M}$, vanishes on horizontal vectors, and obeys the normalisation condition $\Omega(\widehat{w})=1$. In local coordinates

$\mathbf{X}=X^{i}(x) \partial_{i} \mapsto \widehat{\mathbf{X}}=X^{i}(x) \partial_{i}+\gamma_{i}(x) X^{i}(x) \widehat{w}, \quad \Omega=t^{-1} d t-\gamma_{i}(x) d x^{i}, \quad(\Omega(\widehat{w})=1, \Omega(\widehat{\mathbf{X}})=0)$

In particular a volume form $\boldsymbol{\rho}=\rho(x)|D x|$ defines a flat connection:

$$
\nabla_{\boldsymbol{\rho}}: \quad \Omega=d(\log \boldsymbol{\rho}), \gamma_{i}=-\partial_{i} \log \rho(x) .
$$

Now return to the self-adjoint operator $\widehat{\Delta}_{\widehat{S}}$ (see equations (16) and (17)). The principal symbol $\widehat{S}$ of the canonical operator $\widehat{\Delta}_{\widehat{S}}$ and the connection form $\Omega$, which is a covector in the extended manifold $\widehat{M}$, define the vector field $\widehat{S} \Omega$ on $\widehat{M}$ :

$$
\widehat{S} \Omega=\left(\begin{array}{cc}
S^{i k}(x) & B^{i}(x) t \\
B^{k}(x) t & C(x) t^{2}
\end{array}\right)\left(\begin{array}{c}
-\gamma_{k}(x) \\
t^{-1}
\end{array}\right)=\left(B^{i}(x)-S^{i k}(x) \gamma_{k}(x)\right) \partial_{i}+\left(C(x)-B^{i}(x) \gamma_{i}(x)\right) \widehat{w} .
$$

Consider the projection of this vector field to the manifold $M$ :

$$
\text { Projection of vector field } \widehat{S} \Omega \text { on } M=\left(B^{i}(x)-S^{i k}(x) \gamma_{k}(x)\right) \partial_{i} \text {. }
$$

Performing these Kaluza-Klein like considerations (see also [5]) we see that $B^{i}(x)-S^{i k} \gamma_{k}(x)$ is a vector field, i.e. $B^{i}(x)$ is an upper connection (see [6], [7] for details).

In the case when $B^{i}(x)$ belongs to the image of $S^{i k}$ one can impose the condition that the vector field (21) vanishes. In the case that $S^{i k}$ is non-degenerate then this condition uniquely defines a connection such that $B^{i}=S^{i k}(x) \gamma_{k}(x)$. This means that the upper connection $B^{i}(x)=S^{i k}(x) \gamma_{k}(x)$ is generated by a canonical connection. In this case the vector field $\widehat{S} \Omega$ is vertical, i.e. $C(x)-\gamma_{i}(x) S^{i k} \gamma_{k}(x)$ is a scalar field.

In the general case we need more considered analysis. Let $\nabla$ be an arbitrary connection on the fibre bundle $\widehat{M} \rightarrow M$. Consider the following second order operator defined via the connection $\nabla$ and the contravariant tensor field $S^{i k}(x)$ :

$$
\widehat{\Delta}_{\mathbf{S}, \nabla}(s)=\operatorname{div}_{\nabla}\left(\mathbf{S} D_{\nabla} s\right) .
$$

(Compare this operator with operator (16)). Here $D_{\nabla} s$ is a density valued 1-form, a weighted covector on the manifold $M, D_{\nabla} \boldsymbol{s}=\nabla_{i} \boldsymbol{s}(x, t) d x^{i}=\left(\partial_{i} \boldsymbol{s}(x, t)+\widehat{w} \Gamma_{i}(x) \boldsymbol{s}(x, t)\right) d x^{i}$, where $\Gamma_{i}(x)$ are components of the connection $\nabla ; \mathbf{S} D_{\nabla} \boldsymbol{s}=S^{i k}(x, t) \nabla_{k} \boldsymbol{s}(x, t)$ is the corresponding weighted vector field, and $\operatorname{div}_{\nabla}$ is divergence with respect to the connection $\nabla$ : for an arbitrary vector field-density $\mathbf{X}(x, t)=X^{i}(x, t) \partial_{i}$

$$
\operatorname{div}_{\nabla} \mathbf{X}(x, t)=\operatorname{div} \widehat{\mathbf{X}}(x, t)=\partial_{i} X^{i}(x, t)+(\widehat{w}-1) \Gamma_{i}(x) X^{i}(x, t),
$$

where $\widehat{\mathbf{X}}$ is a horizontal lifting of the vector field $\mathbf{X}$ and div is the canonical divergence (9). We come to the answer:

$$
\widehat{\Delta}_{\mathbf{S}, \nabla}=S^{i k}(x) \partial_{i} \partial_{k}+\partial_{r} S^{r i}(x) \partial_{i}+(2 \widehat{w}-1) \Gamma^{i}(x) \partial_{i}+\widehat{w} \partial_{i} \Gamma^{i}(x)+\widehat{w}(\widehat{w}-1) \Gamma^{i} \Gamma_{i}, \quad\left(\Gamma^{i}=S^{i k} \Gamma_{k}\right) .
$$

Comparing this expression with expression (17) for operator (16) we see that

$$
\widehat{\Delta}_{\mathbf{S}, \nabla}=\widehat{\Delta}_{\widehat{S}_{\nabla}}, \quad \text { where } \quad \widehat{S}_{\nabla}=S_{\nabla}(x, t)=\left(\begin{array}{cc}
S^{i k}(x) & \Gamma^{i}(x) t \\
\Gamma^{k}(x) t & \Gamma^{i}(x) \Gamma_{i}(x) t^{2}
\end{array}\right), \quad \Gamma^{i}(x)=S^{i k}(x) \Gamma_{k}(x) .
$$


(Here $\widehat{S}_{\nabla}$ can be considered as a horizontal lifting of $\mathbf{S}$.) One can see that the difference between the operators $\widehat{\Delta}_{\widehat{S}}$ and $\widehat{\Delta}_{\widehat{S}_{\nabla}}$ is equal to $\widehat{\Delta}_{\widehat{S}}-\widehat{\Delta}_{\widehat{S}_{\nabla}}=$

$=(2 \widehat{w}-1) \widehat{\mathcal{L}}_{\mathbf{Y}}+\widehat{w}(\widehat{w}-1)\left(C(x)-2 \Gamma_{i}(x) B^{i}(x)+\Gamma_{i}(x) \Gamma^{i}(x)-\operatorname{div}_{\nabla} \mathbf{Y}\right), Y^{i}=B^{i}-S^{i k} \Gamma_{k}$.

We see that for the operator (17),$Y^{i}=B^{i}(x)-S^{i k} \Gamma_{k}(x)$ is a vector field and $C(x)$ is an object such that $C(x)-2 \Gamma_{i}(x) B^{i}(x)+\Gamma_{i}(x) \Gamma^{i}(x)$ is a scalar function. This is in accordance with transformation laws (19).

Remark 5. Above we analyzed the relation between second order self-adjoint operators on $\widehat{M}$ and their principal symbols. In the article [6] self-adjoint operators were studied with the use of a 'long bracket' which can be assigned to this operator. The long bracket $\{,\}_{\widehat{\Delta}}$ on the space of densities is equal to

$$
\{f(x, t), g(x, t)\}_{\widehat{\Delta}}=\widehat{\Delta}(f g)-f \widehat{\Delta} g-g \widehat{\Delta} f,
$$

(if the normalisation condition $\widehat{\Delta}(1)=0$ holds). The long bracket is equivalent to the principal symbol, and it appears naturally when considering the relation between odd Poisson brackets and odd second order operators on odd symplectic supermanifolds (see [6] and also [7]).

Recall that the problem which we discuss in this article is to construct a pencil lifting of operators on densities of a given weight. In this section we consider pencil liftings of second order operators using the constructions above. Pick an arbitrary $\lambda$ and consider the space $\mathcal{D}_{\lambda}^{(2)}(M)$ of operators of order $\leqslant 2$ acting on densities of weight $\lambda$. Consider an arbitrary operator

$$
\Delta=A^{i k}(x) \partial_{i} \partial_{k}+A^{i}(x) \partial_{i}+A(x)
$$

in $\mathcal{D}_{\lambda}^{(2)}(M)$. (We consider as always only operators of weight $\delta=0$.) Comparing this operator with the operator (16) restricted to $\widehat{w}=\lambda$, and using Proposition 1 we come to

Theorem 1. If $\lambda \neq 0, \frac{1}{2}, 1$ then for an arbitrary operator $\Delta \in \mathcal{D}_{\lambda}^{(2)}(M)$ there exists a unique self-adjoint operator $\widehat{\Delta} \in D^{(2)}(\widehat{M})$ such that

- it obeys normalisation condition (18),

$$
\widehat{\Delta}(1)=0 \text {. }
$$

- it passes through the operator $\Delta$

$$
\left.\widehat{\Delta}\right|_{\widehat{w}=\lambda}=\Delta \text {. }
$$

This operator is equal to the operator $\widehat{\Delta}_{\widehat{S}}$ (see equations (16) and (17)) where $\widehat{S}$ is defined as follows: for the operator $\Delta$ defined by equation (23), $\widehat{S}$ is given by equations

$$
\begin{gathered}
S^{i k}(x)=A^{i k}(x), B^{i}(x)=\frac{A^{i}(x)-\partial_{k} A^{k i}(x)}{2 \lambda-1} \\
C(x)=\frac{A(x)-\lambda \partial_{k} B^{k}(x)}{\lambda(\lambda-1)}=\frac{A(x)}{\lambda(\lambda-1)}-\frac{\partial_{k} A^{k}(x)-\partial_{i} \partial_{k} A^{k i}(x)}{(\lambda-1)(2 \lambda-1)} .
\end{gathered}
$$


This theorem, which was first formulated in [6], allows us to construct a regular pencil lifting map on the spaces $\mathcal{D}_{\lambda}^{(2)}(M)$ assigning to every operator $\Delta \in \mathcal{D}_{\lambda}^{(2)}(M)$ a self-adjoint operator $\widehat{\Delta}=\widehat{\Pi}(\Delta)$ obeying the normalisation condition: if $\Delta=A^{i k}(x) \partial_{i} \partial_{k}+A^{i}(x) \partial_{i}+A(x)$, then $\widehat{\Pi}_{\lambda}(\Delta)$ is given by equation (17) with $S^{i k}(x), B^{i}(x)$ and $C(x)$ defined by equations (24). We come to

$$
\begin{gathered}
\widehat{\Pi}_{\lambda}\left(A^{i k}(x) \partial_{i} \partial_{k}+A^{i}(x) \partial_{i}+A(x)\right)=A^{i j}(x) \partial_{i} \partial_{j}-\frac{2(\widehat{w}-\lambda)}{2 \lambda-1} \partial_{k} A^{k i}(x) \partial_{i}+\frac{2 \widehat{w}-1}{2 \lambda-1} A^{i} \partial_{i}- \\
-\frac{\widehat{w}(\widehat{w}-\lambda)}{(\lambda-1)(2 \lambda-1)}\left(\partial_{i} A^{i}-\partial_{i} \partial_{k} A^{k i}(x)\right)+\frac{\widehat{w}(\widehat{w}-1)}{\lambda(\lambda-1)} A(x), \quad\left(\lambda \neq 0, \frac{1}{2}, 1\right) .
\end{gathered}
$$

The theorem implies that this map is a regular pencil lifting map which is equivariant with respect to arbitrary diffeomorphisms of $M$.

One can consider the restriction of the image of this map to densities of an arbitrary weight $\mu$ :

$$
\Pi_{\lambda}^{\mu}=\left.\widehat{\Pi}_{\lambda}\right|_{\widehat{w}=\mu}
$$

The maps $\Pi_{\lambda}^{\mu}: \mathcal{D}_{\lambda}^{(2)}(M) \rightarrow \mathcal{D}_{\mu}^{(2)}(M)$ are equivariant with respect to the group of diffeomorphisms, i.e. they are maps between $\operatorname{Diff}(M)$-modules $\mathcal{D}_{\lambda}^{(2)}(M)$ and $\mathcal{D}_{\mu}^{(2)}(M)$. In the case if $\lambda, \mu \neq 0, \frac{1}{2}, 1$ these maps establish isomorphsims between these modules. Explicit expressions for these isomorphisms immediately follow from equation (25): If the operator $\Delta=A^{i j}(x) \partial_{i} \partial_{j}+A^{i}(x) \partial_{i}+A(x)$ then its image is the operator $\Pi_{\lambda}^{\mu}(\Delta) \in \mathcal{D}_{\mu}^{(2)}(M)$ given in the same local coordinates by the expression $\Delta_{\mu}=B^{i j}(x) \partial_{i} \partial_{j}+B^{i}(x) \partial_{i}+B(x)$, where

$$
\left\{\begin{array}{l}
B^{i j}=A^{i j} \\
B^{i}=\frac{2 \mu-1}{2 \lambda-1} A^{i}+\frac{2(\lambda-\mu)}{2 \lambda-1} \partial_{j} A^{j i} \\
B=\frac{\mu(\mu-1)}{\lambda(\lambda-1)} A+\frac{\mu(\lambda-\mu)}{(2 \lambda-1)(\lambda-1)}\left(\partial_{j} A^{j}-\partial_{i} \partial_{j} A^{i j}\right)
\end{array}\right.
$$

Remark 6. The theorem provides geometrical background to these isomorphisms which first appeared in the work [4] of Duval and Ovsienko, where they considered the general problem about existence of diff $(M)$-isomorphisms beetween spaces of second order differential operators on densities. The authors of this work described all such isomorphisms. Later it was observed in the article [7] that these isomorphisms can be described by the canonical self-adjoint pencil constructed in [6].

It is illuminating to consider the following example.

Let $\mathbf{X}, \mathbf{Y}$ be two vector fields on a manifold $M$. Consider the second order operator $\Delta=\mathcal{L}_{\mathbf{X}} \mathcal{L}_{\mathbf{Y}} \in \mathcal{D}_{\lambda}^{(2)}$ where $\mathcal{L}_{\mathbf{X}} \mathcal{L}_{\mathbf{Y}}$ are Lie derivatives of densities of weight $\lambda$. We wish to calculate the image of this operator under the isomorphism $\Pi_{\lambda}^{\mu}$. Instead of using formulae (26) directly, we consider a self-adjoint pencil passing through this operator and its restriction to densities of weight $\mu$. The operator $\widehat{\mathcal{L}}_{\mathbf{X}} \circ \widehat{\mathcal{L}}_{\mathbf{Y}}$ defines a pencil of operators which passes through the operator $\Delta=\mathcal{L}_{\mathbf{X}} \circ \mathcal{L}_{\mathbf{Y}}$, but this operator is not self-adjoint. Instead consider the operator:

$$
\widehat{\Delta}=\frac{1}{2}\left(\widehat{\mathcal{L}}_{\mathbf{X}} \circ \widehat{\mathcal{L}}_{\mathbf{Y}}+\widehat{\mathcal{L}}_{\mathbf{Y}} \circ \widehat{\mathcal{L}}_{\mathbf{X}}\right)+\frac{1}{2} \frac{2 \widehat{w}-1}{2 \lambda-1}\left(\widehat{\mathcal{L}}_{\mathbf{X}} \circ \widehat{\mathcal{L}}_{\mathbf{Y}}-\widehat{\mathcal{L}}_{\mathbf{Y}} \circ \widehat{\mathcal{L}}_{\mathbf{X}}\right)
$$


This operator is evidently self-adjoint operator since $\widehat{\mathcal{L}}_{\mathbf{X}} \circ \widehat{\mathcal{L}}_{\mathbf{Y}}+\widehat{\mathcal{L}}_{\mathbf{Y}} \circ \widehat{\mathcal{L}}_{\mathbf{X}}$ is self-adjoint operator and operators $\widehat{\mathcal{L}}_{\mathbf{X}} \circ \widehat{\mathcal{L}}_{\mathbf{Y}}-\widehat{\mathcal{L}}_{\mathbf{Y}} \circ \widehat{\mathcal{L}}_{\mathbf{X}}$ and $2 \widehat{w}-1$ are anti-self adjoint. On the other hand it defines a pencil which passes through the operator $\Delta:\left.\widehat{\Delta}\right|_{\widehat{w}=\lambda}=\mathcal{L}_{\mathbf{X}} \circ \mathcal{L}_{\mathbf{Y}}$ and normalisation condition is evidently obeyed. The theorem implies that this formula defines the image of the operator $\Delta=\mathcal{L}_{\mathbf{X}} \circ \mathcal{L}_{\mathbf{Y}}$ under the isomorphism $\Pi_{\lambda}^{\mu}$ :

$$
\begin{gathered}
\Pi_{\lambda}^{\mu}(\Delta)=\left.\widehat{\Delta}\right|_{\widehat{w}=\mu}=\frac{1}{2}\left(\mathcal{L}_{\mathbf{X}} \circ \mathcal{L}_{\mathbf{Y}}+\mathcal{L}_{\mathbf{Y}} \circ \mathcal{L}_{\mathbf{X}}\right)+\frac{1}{2} \frac{2 \mu-1}{2 \lambda-1}\left(\mathcal{L}_{\mathbf{X}} \circ \mathcal{L}_{\mathbf{Y}}-\mathcal{L}_{\mathbf{Y}} \circ \mathcal{L}_{\mathbf{X}}\right)= \\
\mathcal{L}_{\mathbf{X}} \circ \mathcal{L}_{\mathbf{Y}}+\frac{\mu-\lambda}{2 \lambda-1} \mathcal{L}_{[\mathbf{X}, \mathbf{Y}]} .
\end{gathered}
$$

Remark 7. This example first appeared in [11] where it was calculated using a different technique.

\section{Pencil Liftings equivariant with Respect to sdiff $(M)$}

Now consider pencil liftings for the space of differential operators of an arbitrary order. We would like to consider pencil liftings $\mathcal{D}_{\lambda}^{(n)} \ni \Delta \rightarrow \widehat{\Pi}(\Delta)$ for arbitrary $n$. In general there is no $\operatorname{diff}(M)$-equivariant pencil liftings (see [9], [11] and [2]). We consider pencil liftings which are equivariant with respect to the smaller algebra, sdiff $(M)$, of divergenceless vector fields.

Let $\boldsymbol{\rho}=\rho(x)|D x|$ be a volume form on $M$. It defines a group SDiff $\boldsymbol{\rho}(M)$ of volume preserving diffeomorphisms and the corresponding Lie algebra sdiff $\rho(M)$.

A volume form structure on the manifold $M$ allows one to identify spaces of densities of different weights and operators on them by the following canonical isomorphisms

$$
\begin{gathered}
\mathcal{F}_{\lambda}(M) \ni \boldsymbol{s}(x) \rightarrow \boldsymbol{s}^{\prime}(x)=\boldsymbol{s}(x) \boldsymbol{\rho}^{\mu-\lambda} \in \mathcal{F}_{\mu}(M), \\
P_{\lambda}^{\mu}: \quad \mathcal{D}_{\lambda}^{(n)}(M) \ni \Delta \rightarrow \Delta^{\prime}=P_{\lambda}^{\mu}(\Delta)=\boldsymbol{\rho}^{\mu-\lambda} \circ \Delta \circ \boldsymbol{\rho}^{\lambda-\mu} \in \mathcal{D}_{\mu}^{(n)}(M) .
\end{gathered}
$$

It is useful to write down explicit formulae for isomorphisms $P_{\lambda}^{\mu}$ in local coordinates. If $\Delta=\sum_{k=0}^{n} S^{i_{1} \ldots i_{k}}(x) \partial_{i_{1}} \ldots \partial_{k}$ is an operator of order $\leqslant n$ acting on densities of weight $\lambda$, then

$$
\begin{gathered}
P_{\lambda}^{\mu}(\Delta)=\sum_{k=0}^{n} \rho^{\mu-\lambda}(x) \circ S^{i_{1} \ldots i_{k}}(x) \partial_{i_{1}} \ldots \partial_{i_{k}} \circ \rho^{\lambda-\mu}(x)= \\
S^{i_{1} \ldots i_{k}}(x)\left(\partial_{i_{1}}+(\mu-\lambda) \Gamma_{i_{1}}\right) \ldots\left(\partial_{i_{k}}+(\mu-\lambda) \Gamma_{i_{k}}\right),
\end{gathered}
$$

where $\Gamma_{i}=-\partial_{i} \log \rho_{i}$ are components of the flat connection induced by the volume form $\boldsymbol{\rho}=\rho(x)|D x|$ (see equation (20)). This formula follows from the observation that $\left[\partial_{i}, \rho^{\lambda}\right]=$ $\partial_{i} \circ \rho^{\lambda}-\rho^{\lambda} \circ \partial_{i}=-\lambda \Gamma_{i}(x)$.

On a manifold with a volume form structure the isomorphisms $\left\{P_{\lambda}^{\mu}\right\}$ define a canonical pencil lifting $\widehat{P}_{\lambda}$ on operators acting on densities of weight $\lambda$ :

$$
\widehat{P}_{\lambda}:\left.\quad \widehat{P}_{\lambda}\right|_{\widehat{w}=\mu}=P_{\lambda}^{\mu}, \quad\left(\widehat{P}_{\lambda}(\Delta)=\boldsymbol{\rho}^{\widehat{w}-\lambda} \circ \Delta \circ \boldsymbol{\rho}^{\lambda-\widehat{w}}\right) .
$$

If $\Delta=\sum S^{i_{1} \ldots i_{k}}(x) \partial_{i_{1}} \ldots \partial_{k}$, then

$$
\widehat{P}_{\lambda}(\Delta)=\sum S^{i_{1} \ldots i_{k}}(x)\left(\partial_{i_{1}}+(\widehat{w}-\lambda) \Gamma_{i_{1}}\right) \ldots\left(\partial_{i_{k}}+(\widehat{w}-\lambda) \Gamma_{i_{k}}\right) .
$$


It is evident that this canonical pencil lifting defined on operators of an arbitrary order is equivariant with respect to the Lie algebra sdiff ${ }_{\rho}(M)$ and the group $\operatorname{SDiff}_{\rho}(M)$. We now search for other pencil liftings which are equivariant with respect to this algebra and to this group. Using these isomorphisms we may reduce the problem of constructing of sdiff $(M)$ equivariant pencil liftings to finding certain maps between operators on functions (densities of weight $\lambda=0$ ).

Namely let $F=F(\Delta)$ be a linear map on the space of operators on functions $F: \mathcal{D}_{0}^{(n)}(M) \rightarrow$ $\mathcal{D}_{0}^{(n)}(M)$. Then using the canonical isomorphisms one can assign to this map the map $\widehat{\Pi}^{(F)}$, defined on operators on $\mathcal{F}_{\lambda}(M)$ with values in operators on the algebra of densities: if $\Delta$ is an operator on $\mathcal{F}_{\lambda}(M)$ then

$$
\widehat{\Pi}_{\lambda}^{(F)}(\Delta)=\boldsymbol{\rho}^{\widehat{w}} \circ F(\underbrace{\boldsymbol{\rho}^{-\lambda} \circ \Delta \circ \boldsymbol{\rho}^{\lambda}}_{\text {operator on functions }}) \circ \boldsymbol{\rho}^{-\widehat{w}}
$$

(We denote by $\boldsymbol{\rho}^{\widehat{w}} \circ \Delta \circ \boldsymbol{\rho}^{-\widehat{w}}$ the operator $\widehat{\Delta}$ such that for an arbitrary weight $\mu,\left.\widehat{\Delta}\right|_{\widehat{w}=\mu}=$ $\boldsymbol{\rho}^{\mu} \circ \Delta \circ \boldsymbol{\rho}^{-\mu}$.)

The condition that the pencil $\widehat{\Pi}_{\lambda}^{(F)}(\Delta)$ passes through the operator $\Delta,\left.\widehat{\Pi}(\Delta)\right|_{\widehat{w}=\lambda}=\Delta$ reads that

$$
\boldsymbol{\rho}^{\lambda} \circ F\left(\rho^{-\lambda} \circ \Delta \circ \rho^{\lambda}\right) \circ \rho^{-\lambda}=\Delta
$$

The map $\widehat{\Pi}^{(F)}$ is sdiff $(M)$-equivariant iff the map $F$ is sdiff $(M)$-equivariant and this condition is obeyed.

Consider the following map $F$ on operators on functions

$$
F(\Delta)=\alpha \Delta+\beta \Delta^{* \rho}+\gamma \Delta(1)+\delta \Delta^{* \rho}(1) .
$$

Here $\alpha, \beta, \gamma$, and $\delta$ are constant coefficients and $\Delta^{*} \rho$ is the operator on functions which is adjoint to the operator $\Delta$ with respect to the volume form $\boldsymbol{\rho}: \int_{M} \Delta f \cdot g \boldsymbol{\rho}=\int_{M} f \cdot\left(\Delta^{*} g\right) \boldsymbol{\rho}$ for two arbitrary functions $f, g$ with compact support. (Compare with the definition of the canonical adjoint (see after equation (30).) It is evident that this map is equivariant with respect to all diffeomorphisms preserving the volume form and with respect to all divergenceless vector fields. Simple calculations show that the corresponding map $\widehat{\Pi}^{(F)}(\Delta)$ (see equation (28)) on operators on $\mathcal{F}_{\lambda}(M)$ is equal to:

$$
\widehat{\Pi}_{\lambda}^{(F)}(\Delta)=a \widehat{P}_{\lambda}(\Delta)+b\left(\widehat{P}_{\lambda}(\Delta)\right)^{*}+c\left(\widehat{P}_{\lambda}(\Delta)\right)(1)+d\left(\widehat{P}_{\lambda}(\Delta)\right)^{*}(1)
$$

where $\widehat{P}_{\lambda}$ is the pencil lifting (27) and (*) denotes the canonical adjoint.

Now choose an arbitrary $n$ and an arbitrary weight $\lambda$, then using the last equation, we will write down a family of regular sdiff $\rho(M)$-pencil liftings on the space of operators of order $\leqslant n$ on densities of weight $\lambda$. Bearing in mind condition (29) and the fact that the pencil lifting is regular, (i.e. the order of the operator $\widehat{\Pi}(\Delta)$ cannot be greater than $n$ if the order of the operator $\Delta$ is not greater than $n$ ), we come to the fact that the coefficients $\alpha, \beta, \gamma, \delta$ 
are polynomials in the weight operator $\widehat{w}$, i.e. vertical operators such that

$$
\left\{\begin{array}{l}
\alpha=A(\widehat{w}), \beta=B(\widehat{w}) \text { have order } \leqslant 1 \text { in } \widehat{w} \\
\gamma=C(\widehat{w}), \delta=D(\widehat{w}) \text { have order } \leqslant n \text { in } \widehat{w} \\
A(\widehat{w})+(-1)^{n} B(\widehat{w})=1, \\
\left.A(\widehat{w})\right|_{\widehat{w}=\lambda}=1,\left.B(\widehat{w})\right|_{\widehat{w}=\lambda}=0 \\
\left.C(\widehat{w})\right|_{\widehat{w}=\lambda}=\left.D(\widehat{w})\right|_{\widehat{w}=\lambda}=0
\end{array}\right.
$$

the regularity condition of the pencil and the condition that the pencil passes through the operator

Thus we come to the following family of regular sdiff $\rho^{- \text {equivariant (and SDiff } \rho} \boldsymbol{\rho}(M)$-equivariant) pencil liftings: $\widehat{\Pi}_{\lambda}$, such that for an arbitrary $\Delta \in \mathcal{D}_{\lambda}^{(n)}(M)$,

$$
\widehat{\Pi}_{\lambda}(\Delta)=A(\widehat{w}) \widehat{P}_{\lambda}(\Delta)+B(\widehat{w})\left(\widehat{P}_{\lambda}(\Delta)\right)^{*}+C(\widehat{w})\left(\widehat{P}_{\lambda}(\Delta)\right)(1)+D(\widehat{w})\left(\widehat{P}_{\lambda}(\Delta)\right)^{*}(1),
$$

where

$A(\widehat{w})=1-b(\widehat{w}-\lambda), B(\widehat{w})=(-1)^{n} b(\widehat{w}-\lambda), C(\widehat{w})=\sum_{k=1}^{n} c_{k}(\widehat{w}-\lambda)^{k}, D(\widehat{w})=\sum_{k=1}^{n} d_{k}(\widehat{w}-\lambda)^{k}$,

$b, c_{k}, d_{k}$ are arbitrary coefficients (see also [2]). We come to a $2 n+1$-dimensional plane of regular pencil liftings. The following lemma (see its proof in appendix to the article [2]) shows that this family of pencil liftings exhausts all regular sdiff $(M)$-equivariant liftings (if the manifold is of dimension greater than 2).

Lemma 1. Let $F$ be a local linear map on the space $\mathcal{D}_{0}^{(n)}(M)$ of differential operators of order $\leqslant n$ on functions which is equivariant with respect to the Lie algebra $\operatorname{sdiff}_{\rho}(M)$ of divergenceless vector fields. If the manifold $M$ is connected and of dimension greater than 2 , then $F$ is expressed by equation (30).

(One should note that local linear maps are differential polynomials on the coefficients of operators (see Peetre's theorem [13]).)

Example 4.1. In this example we consider sdiff $(M)$-equivariant pencil liftings of $\mathcal{D}_{\lambda}^{(1)}\left(\mathbb{R}^{n}\right)$. Let $\boldsymbol{\rho}$ be a volume form in Cartesian coordinates, $\boldsymbol{\rho}=\rho(x)|D x|$. Denote by $\Gamma_{i}(x)=$ $-\partial_{i} \log \rho(x)$ the components of the flat connection induced by the volume form (see (20)). If $\Delta=X^{i}(x) \partial_{i}+F(x)$ is an arbitrary first order operator acting on densities of weight $\lambda$, then we see that according to equations (27)

$$
\begin{gathered}
\widehat{P}_{\lambda}(\Delta)=X^{i}(x)\left(\partial_{i}+(\widehat{w}-\lambda) \Gamma_{i}(x)\right)+F(x), \\
\widehat{P}_{\lambda}^{*}(\Delta)=-X^{i}(x) \partial_{i}-\partial_{i} X^{i}(x)+(1-\widehat{w}-\lambda) X^{i}(x) \Gamma_{i}(x)+F(x), \\
\widehat{P}_{\lambda}(\Delta)(1)=\left(X^{i}(x)\left(\partial_{i}+(\widehat{w}-\lambda) \Gamma_{i}(x)\right)+F(x)\right)(1)=-\lambda X^{i}(x) \Gamma_{i}(x)+F(x), \\
\widehat{P}_{\lambda}^{*}(\Delta)(1)=-\partial_{i} X^{i}(x)+(1-\lambda) X^{i}(x) \Gamma_{i}(x)+F(x) .
\end{gathered}
$$

Thus according to (31) we come to the following family of regular sdiff $(M)$-pencil liftings:

$$
\begin{gathered}
\widehat{\Pi}_{\lambda}(\Delta)=X^{i}(x) \partial_{i}+b(\widehat{w}-\lambda) \partial_{i} X^{i}(x)+(\widehat{w}-\lambda)(1-b(2 \lambda-1)) X^{i}(x) \Gamma_{i}(x)+(1-2 b(\widehat{w}-\lambda)) F(x)+ \\
+c(\widehat{w}-\lambda)\left(F(x)-X^{i}(x) \Gamma_{i}(x)\right)+d(\widehat{w}-\lambda)\left(F(x)-\partial_{i} X^{i}(x)+(1-\lambda) X^{i}(x) \Gamma_{i}(x)\right),
\end{gathered}
$$

where $b, c, d$ are constants. 
Now we analyse the geometrical meaning of the family (31) of pencil liftings. One can choose a line of liftings

$$
\left\{\widehat{\Pi}_{\lambda}^{b}\right\}: \quad \widehat{\Pi}_{\lambda}^{b}(\Delta)=(1-b(\widehat{w}-\lambda)) \widehat{P}_{\lambda}(\Delta)+(-1)^{n} b(\widehat{w}-\lambda)\left(\widehat{P}_{\lambda}(\Delta)\right)^{*}, b \in \mathbb{R} .
$$

An arbitrary lifting as in (31), $\widehat{\Pi}_{\lambda}$, is the sum of a lifting $\widehat{\Pi}^{b}$ for some $b$, and a map which takes values in vertical operators. Notice that in the case if $\lambda \neq \frac{1}{2}$ then the condition

$$
b=\frac{1}{1-2 \lambda}
$$

makes the pencil lifting (32) self-adjoint if $n$ is even and anti-self-adjoint if $n$ is odd. This follows from the fact that the vertical operators $1-b(\widehat{w}-\lambda)$ and $b(\widehat{w}-\lambda)$ are the adjoints of each other iff condition (33) is obeyed:

$$
1-b(\widehat{w}-\lambda)=(b(\widehat{w}-\lambda))^{*}=b(1-\widehat{w})-b \lambda \Leftrightarrow b=\frac{1}{1-2 \lambda} .
$$

Consider the pencil lifting (32) for $b=\frac{1}{1-2 \lambda}$ : We will call this pencil lifting distinguished and denote it $\widehat{\Pi}_{\lambda}^{\text {disting }}$

$$
\begin{gathered}
\widehat{\Pi}_{\lambda}^{\text {disting }}(\Delta)=\left.\widehat{\Pi}_{\lambda}^{b}(\Delta)\right|_{b=\frac{1}{1-2 \lambda}}=\frac{\widehat{w}+\lambda-1}{2 \lambda-1} \widehat{P}_{\lambda}(\Delta)+(-1)^{n} \frac{\lambda-\widehat{w}}{2 \lambda-1}\left(\widehat{P}_{\lambda}(\Delta)\right)^{*}, \\
\left(\widehat{\Pi}_{\lambda}^{\text {disting }}(\Delta)\right)^{*}=(-1)^{n} \widehat{\Pi}_{\lambda}^{\text {disting }}(\Delta) .
\end{gathered}
$$

Comparing the family of pencil liftings (31) with the line of liftings (32) and with the distinguished pencil lifting we see that that for every sdiff ${ }_{\rho}(M)$-pencil lifting $\widehat{\Pi}_{\lambda}$ the following decomposition holds:

$$
\begin{aligned}
& \text { for all } \Delta \in \mathcal{D}_{\lambda}^{(n)}(M), \widehat{\Pi}_{\lambda}(\Delta)=(1-b(\widehat{w}-\lambda)) \widehat{P}_{\lambda}(\Delta)+(-1)^{n} b(\widehat{w}-\lambda) \widehat{P}_{\lambda}^{*}(\Delta)+\cdots= \\
& \qquad \widehat{\Pi}_{\lambda}^{b}(\Delta)+\cdots= \\
& =\widehat{\Pi}_{\lambda}^{\text {disting }}(\Delta)-k(\widehat{w}-\lambda)\left(\widehat{P}_{\lambda}(\Delta)-(-1)^{n} \widehat{P}_{\lambda}^{*}(\Delta)\right)+\ldots, \text { where } k=b-\frac{1}{1-2 \lambda}, \quad
\end{aligned}
$$

and the ellipses mean vertical operators. It is useful at this step to introduce the following notation: Recall that operator an $L \in \mathcal{D}(M)$ is vertical if it commutes with functions on $M, L=L(t, x, \widehat{w})$. We denote by $\mathcal{V}^{(0)}(M)$ the space of vertical operators on the algebra of densities. Inductively we define $\mathcal{V}^{(n)}(M)$ as follows: an operator $\Delta$ on $\mathcal{F}(M)$ is in $\mathcal{V}^{(n)}(M)$ if for an arbitrary function $f$ on $M,[\Delta, f]$ is in $\mathcal{V}^{(n-1)}(M)$. Roughly speaking operators in $\mathcal{V}^{(n)}(M)$ are differential operators of order $\leqslant n$ with coefficients which are vertical operators. For example consider the second term in the equation (35), the operator $\widehat{\Delta}=k(\widehat{w}-\lambda)\left(\widehat{P}_{\lambda}(\Delta)-(-1)^{n} \widehat{P}_{\lambda}^{*}(\Delta)\right)$. It belongs to the space $\mathcal{D}^{n}(\widehat{M})$ of operators of order $\leqslant n$ on the algebra of densities $\mathcal{F}(M)$, but as this operator has spatial derivatives of order $\leqslant n-1$, it belongs to the space $\mathcal{V}^{(n-1)}(M)$.

Proposition 2. In the case if $\lambda \neq \frac{1}{2}$, then the family (31) of regular sdiff $(M)$-equivariant pencil liftings of the space $\mathcal{D}_{\lambda}^{(n)}(M)$, contains distinguished pencil lifting (34). This lifting is self-adjoint if $n$ is even and it is anti-self-adjoint if $n$ is odd. The distinguished pencil lifting, which is defined on the space $\mathcal{D}_{\lambda}^{(n)}(M)$ factored by the space $\mathcal{V}^{(n-2)}(M)$ does not depend on 
the volume form. In other words the terms of operator $\widehat{\Pi}^{\mathrm{disting}}(\Delta)$ which possess spatial derivatives of order $n$ and $n-1$ do not depend on the choice of the volume form.

The map to the space $\mathcal{D}^{(n)}(\widehat{M})$ factored by $\mathcal{V}^{(n-2)}(M)$ induced by the distinguished pencil lifting is diff $(M)$-equivariant.

Any map to the factor space that is $\operatorname{diff}(M)$-equivariant and induced by a pencil lifting in (31) must be induced by the distinguished pencil lifting.

This Proposition can be proved directly. Consider an example.

Example 4.2. Consider regular sdiff $(M)$-equivariant pencil liftings of fourth order operators. We will perform all calculations up to the terms containing spatial derivatives of order $\geqslant 3$, i.e. we factor out the space $\mathcal{V}^{(2)}(M)$.

Let $\Delta=S^{i k m n}(x) \partial_{i} \partial_{k} \partial_{m} \partial_{n}+L^{i k m} \partial_{i} \partial_{k} \partial_{m}+\ldots, \Delta \in \mathcal{D}_{\lambda}^{(4)}(M),\left(\lambda \neq \frac{1}{2}\right)$, be an operator of order $\leqslant 4$ acting on densities of weight $\lambda$ on the manifold $M$ provided with a volume form $\boldsymbol{\rho}=\rho(x)|D x|$. As usual denote by $\Gamma_{i}(x)=-\partial_{i} \log \rho(x)$ the components of the flat connection corresponding to this volume form. Then it follows from (27) that

$$
\widehat{P}_{\lambda}(\Delta)=S^{i k m n}(x) \partial_{i} \partial_{k} \partial_{n} \partial_{m}+\left(4(\widehat{w}-\lambda) \Gamma_{r} S^{r i k m}+L^{i k m}(x)\right) \partial_{i} \partial_{k} \partial_{m}+\ldots,
$$

$\widehat{P}_{\lambda}^{*}(\Delta)=S^{i k m n}(x) \partial_{i} \partial_{k} \partial_{n} \partial_{m}+\left(4(\widehat{w}+\lambda-1) \Gamma_{r} S^{r i k m}(x)+4 \partial_{r} S^{r i k m}(x)-L^{i k m}(x)\right) \partial_{i} \partial_{k} \partial_{m}+\ldots$, and using the decomposition formulae (35) we come to

$$
\begin{gathered}
\widehat{\Pi}_{\lambda}(\Delta)=\underbrace{\frac{\widehat{w}+\lambda-1}{2 \lambda-1} \widehat{P}_{\lambda}(\Delta)+\frac{\lambda-\widehat{w}}{2 \lambda-1} \widehat{P}_{\lambda}^{*}(\Delta)}_{\text {operator } \widehat{\Pi}_{\lambda}^{\text {disting }}(\Delta)}+ \\
k(\widehat{w}-\lambda)\left(\widehat{P}_{\lambda}^{*}(\Delta)-\widehat{P}_{\lambda}(\Delta)\right)+\cdots= \\
\underbrace{S^{i k m n}(x) \partial_{i} \partial_{k} \partial_{m} \partial_{n}+\left(\frac{\lambda-\widehat{w}}{2 \lambda-1} 4 \partial_{r} S^{r i k m}(x)+\frac{2 \widehat{w}-1}{2 \widehat{\lambda}-1} L^{i k m}(x)\right) \partial_{i} \partial_{k} \partial_{m}}_{\text {operator } \widehat{\Pi}_{\lambda}^{\text {disting }}(\Delta)}+ \\
k(\widehat{w}-\lambda)\left(4 \partial_{r} S^{\text {rikm }}(x)+4(2 \lambda-1) \Gamma_{r}(x) S^{\text {rikm }}(x)-2 L^{i k m}(x)\right) \partial_{i} \partial_{k} \partial_{m}+\ldots
\end{gathered}
$$

We see that if $k=0$, i.e. $\widehat{\Pi}_{\lambda}=\widehat{\Pi}_{\lambda}^{\text {disting }}$, then the lifting does not depend on the volume form up to $\mathcal{V}^{(2)}(M)$.

Remark 8. Another proof of this proposition is to calculate the variation of liftings (35) with respect to variation of the volume form, using the formula

$$
\delta P_{\lambda}=(\widehat{w}-\lambda)\left[\boldsymbol{\rho}^{-1} \delta \boldsymbol{\rho}, P_{\lambda}\right] .
$$

Then one can see that for an arbitrary operator $\Delta$ the variation of the operator $\widehat{\Pi}^{\text {disting }}(\Delta)$ lies in $\mathcal{V}^{(n-2)}$, it vanishes in the factorspace (see for details [2]).

Regular pencil liftings equivariant with respect to the Lie algebra sdiff $\rho(M)$ can be defined on operators of arbitrary order. The Lie algebra sdiff $\rho(M)$ is in some sense the 'closest' to the Lie algebra diff $(M)$ of all vector fields. It follows from proposition 2 that any diff $(M)$ equivariant pencil lifting must be (anti)self-adjoint up to a simple factor. More precisely 
if a sdiff $(M)$-pencil lifting $\widehat{\Pi}$ is additionally equivariant with respect to the Lie algebra $\operatorname{diff}(M)$ then the pencil lifting $\widehat{\Pi}$ is equal to the distinguished pencil lifting $\widehat{\Pi}^{\text {disting }}$ up to a map with values in vertical operators. This simple remark becomes useful if one studies $\operatorname{diff}(M)$-equivariant pencil liftings for manifolds of dimension greater than 2 , since in this case equation (31) describes all regular sdiff $(M)$ liftings. For example in previous sections we considered canonical pencil liftings for first order operators and the self-adjoint canonical lifting for second order operators. These liftings are evidently diff $(M)$-equivariant. Considering sdiff $(M)$-equivariant distinguished pencil liftings on operators of first and second order and, if necessary correcting them by vertical maps, one can prove that for manifolds of dimension $\geqslant 3$, there are no other diff $(M)$-equivariant liftings except those considered in the previous sections (for details see [2]).

\section{The DLO-Pencil Lifting And proj $(M)$-EQUivariant Liftings}

In this section we consider regular pencil liftings equivariant with respect to the Lie algebra $\operatorname{proj}(M)$ of projective vector fields. Without loss of generality we consider $M=\mathbb{R}^{d}$ with Cartesian coordinates $\left\{x^{i}\right\}=\left\{x^{1}, \ldots, x^{d}\right\}$, the Lie algebra proj $\left(\mathbb{R}^{d}\right)$ is generated by vector fields

$$
\left\{\begin{array}{l}
\text { translations } \partial_{i} \\
\text { linear transformations } x^{k} \partial_{i} \\
\text { special projective transformations } x^{k} x^{i} \partial_{i}
\end{array} \quad i, k=1, \ldots, d .\right.
$$

We will first construct strictly regular DLO-pencil lifting (see equations (7) and (8) in Introduction) on the spaces of operators $\mathcal{D}_{\lambda}^{(n)}\left(\mathbb{R}^{d}\right)$ (for arbitrary $\lambda$ and $n$ ), then using this pencil lifting we will construct all regular $\operatorname{proj}\left(\mathbb{R}^{d}\right)$-pencil liftings on $\mathcal{D}_{\lambda}^{(n)}\left(\mathbb{R}^{d}\right)$.

In the classical articles [10] and [3] of Duval, Lecomte and Ovsienko projective and conformal equivariant quantisation was considered. They constructed full symbol maps equivariant with respect to the Lie algebra of projective transformations [10] and full symbol maps equivariant with respect to the Lie algebra of conformal transformations [3]. These symbol maps were defined on operators acting on densities of arbitrary weight. We consider here the projective case. (The conformal case can be considered analogously.) We consider the full symbol map $\sigma_{\lambda}$ constructed in [10] which is a linear map on operators with values in contravariant symmetric tensor fields (polynomials in the fibre variables of $T^{*} M$ ), obeying the natural normalisation conditions (see ([6) in Introduction.) For an arbitrary operator, $\Delta$,

$$
\begin{gathered}
\mathcal{D}_{\lambda}^{(n)}\left(\mathbb{R}^{d}\right) \ni \Delta=\sum_{k=0}^{n} S^{i_{1} \ldots i_{k}}(x) \partial_{i_{1}} \ldots \partial_{i_{k}} \\
\sigma(\Delta)=\sum_{k=0}^{n} \sum_{r=0}^{k} c_{r}^{(k)}(\lambda) \partial_{j_{1}} \ldots \partial_{j_{r}} S^{j_{1} \ldots j_{r} i_{1} \ldots i_{k-r}} \xi_{i_{1}} \ldots \xi_{i_{k-r}},
\end{gathered}
$$

where the $c_{r}^{(k)}(\lambda)(r=0,1, \ldots, k)$ are polynomials in $\lambda$ of order $r$ obeying the normalisation condition

$$
c_{0}^{(k)}=1 .
$$

The form of the full symbol map is dictated by the equivariance with respect to affine transformations. Equivariance with respect to the special projective transformations defines 
a recurrent relation amongst the polynomials $c_{r}^{(k)}(\lambda)$ (see for detail [10] and the book [12]). E.g.

$$
\begin{gathered}
\sigma\left(S^{i k}(x) \partial_{i} \partial_{k}+A^{i}(x) \partial_{i}+F(x)\right)=S^{i k}(x) \xi_{i} \xi_{k}-\frac{2(\lambda(d+1)+1)}{d+3} \partial_{k} S^{k i}(x) \xi_{i}+ \\
\frac{\lambda(\lambda(d+1)+1)}{d+2} \partial_{k} \partial_{i} S^{k i}(x)+A^{i}(x) \xi_{i}-\lambda \partial_{i} A^{i}(x)+F
\end{gathered}
$$

One can consider a quantisation map $Q_{\lambda}$ which is inverse to the full symbol map, $Q_{\lambda}=\sigma_{\lambda}^{-1}$,

$$
Q_{\lambda}\left(S^{i_{1} \ldots i_{k}} \xi_{1} \ldots \xi_{k}\right)=\sum_{r=0}^{k} \tilde{c}_{r}^{(k)}(\lambda) \partial_{j_{1}} \ldots \partial_{j_{r}} S^{j_{1} \ldots j_{r} i_{1} \ldots i_{k-r}} \partial_{i_{1}} \partial_{i_{2}} \ldots \partial_{i_{k-r}}
$$

where $\tilde{c}_{r}^{(k)}$ are polynomials in $\lambda$ which can be expressed recurrently through the polynomials $c_{k}(\lambda)$ by the formulae $\tilde{c}_{0}^{(k)} c_{0}^{(k)}=1$ and $\sum_{i=0}^{p} \tilde{c}_{p-i}^{(k)} c_{i}^{(k-p+i)}=0$ if $p>0$. We come to $\tilde{c}_{0}^{(k)}=1$, $\tilde{c}_{1}^{k}(\lambda)=-c_{1}^{(k)}(\lambda), \tilde{c}_{2}^{(k)}(\lambda)=-c_{2}^{(k)}(\lambda)-\tilde{c}_{1}^{(k)}(\lambda) c_{1}^{(k-1)}(\lambda)=-c_{2}^{(k)}(\lambda)+c_{1}^{(k)}(\lambda) c_{1}^{(k-1)}(\lambda), \ldots$ In particular it follows from (36) that

$$
\begin{gathered}
Q_{\lambda}\left(S^{i k}(x) \xi_{i} \xi_{k}+A^{i}(x) \xi_{i}+F(x)\right)=S^{i k}(x) \partial_{i} \partial_{k}+a_{d}(\lambda) \partial_{k} S^{k i}(x) \partial_{i}+ \\
b_{d}(\lambda) \partial_{k} \partial_{i} S^{k i}(x)+A^{i}(x) \partial_{i}+\lambda \partial_{i} A^{i}(x)+F
\end{gathered}
$$

where

$$
a_{d}(\lambda)=\frac{2(\lambda(d+1)+1)}{d+3}, \quad b_{d}(\lambda)=\frac{\lambda(d+1)(\lambda(d+1)+1)}{(d+2)(d+3)} .
$$

As was already explained in the Introduction (see (7D) using the full symbol map $\sigma_{\lambda}$ and the quantisation map $Q_{\lambda}$, one can consider proj $\left(\mathbb{R}^{d}\right)$-pencil lifting $\widehat{\Pi}^{\mathrm{DLO}}$, DLO-pencil lifting:

$$
\widehat{\Pi}_{\lambda}^{\mathrm{DLO}}: \widehat{\Pi}^{\mathrm{DLO}}=\hat{Q}_{\widehat{w}} \circ \sigma_{\lambda},
$$

where $\hat{Q}_{\widehat{w}}:\left.\hat{Q}\right|_{\widehat{w}=\mu}=Q_{\mu},\left(\left.\widehat{\Pi}_{\lambda}^{\mathrm{DLO}}\right|_{\widehat{w}=\mu}=Q_{\mu} \circ \sigma_{\lambda}\right)$. ( It is easy to see that for every operator $\Delta \in \mathcal{D}_{\lambda}^{(n)}, \widehat{\Pi}_{\lambda}^{D L O}(\Delta)$ is the differential operator on the algebra of densities since all the coefficients $c_{r}^{(k)}(\lambda), \tilde{c}_{r}^{(k)}(\lambda)$ are polynomials in $\lambda$.)

One can see that the DLO-pencil lifting is a proj-equivariant strictly regular pencil lifting which can be defined on operators of arbitrary order. Our construction of this pencil lifting is nothing but a reformulation of some of the results due to Duval, Lecomte and Ovsienko. Our next step is to construct a family of proj-equivariant pencils 'dressing' the DLO-pencil lifting with vertical operators. For this purpose we rewrite the DLO-pencil lifting in a different way.

Consider the following canonical decomposition (with respect to a projective structure) of the DLO-pencil lifting. Firstly for an arbitrary $n$ introduce the proj-equivariant linear map $\widehat{\Pi}_{n, \lambda}$ defined on the space $\mathcal{D}_{\lambda}^{(n)}\left(\mathbb{R}^{d}\right)$ of operators of order $\leqslant n$ on densities of weight $\lambda$ :

$$
\widehat{\Pi}_{n, \lambda}: \quad \mathcal{D}_{\lambda}^{(n)} \ni \Delta, \widehat{\Pi}_{n, \lambda}(\Delta)=\hat{Q}_{\widehat{w}}\left(\Sigma_{\text {princ. }}^{(n)}(\Delta)\right),
$$

where $\Sigma_{\text {princ. }}^{(n)}(\Delta)$ is the principal symbol of operator $\Delta$. If $\Delta=S^{i_{1} \ldots i_{n}} \partial_{i_{1}} \ldots \partial_{i_{n}}+\ldots$ then using equation (37) we come to

$$
\widehat{\Pi}_{n, \lambda}(\Delta)=\hat{Q}_{\widehat{w}}\left(S^{i_{1} \ldots i_{n}} \xi_{i_{1}} \ldots \xi_{i_{n}}\right)=\sum \tilde{c}_{r}^{(n)}(\widehat{w}) \partial_{j_{1}} \ldots \partial_{j_{r}} S^{j_{1} \ldots j_{r} i_{1} \ldots i_{n-r}} \partial_{i_{1}} \ldots \partial_{i_{n-r}} .
$$


For example

$$
\begin{gathered}
\widehat{\Pi}_{0, \lambda}(F(x))=F(x) \\
\widehat{\Pi}_{1, \lambda}\left(A^{i}(x) \partial_{i}+F(x)\right)=\hat{Q}_{\widehat{w}}\left(A^{i}(x) \xi_{i}\right)=A^{i}(x) \partial_{i}+\widehat{w} \partial_{i} A^{i}(x), \\
\widehat{\Pi}_{2, \lambda}\left(S^{i k}(x) \partial_{i} \partial_{k}+A^{i}(x) \partial_{i}+F(x)\right)=Q_{\lambda}\left(S^{i k}(x) \xi_{i} \xi_{k}\right)= \\
=S^{i k}(x) \partial_{i} \partial_{k}+a_{d}(\widehat{w}) \partial_{k} S^{k i}(x) \partial_{i}+b_{d}(\widehat{w}) \partial_{i} \partial_{k} S^{i k}(x)
\end{gathered}
$$

where the polynomials $a_{d}, b_{d}$ are defined by equations (39).

One can see that the operator $\Delta-\left.\widehat{\Pi}_{n, \lambda}(\Delta)\right|_{\widehat{w}=\lambda}=\Delta-Q_{\lambda}\left(\Sigma_{\text {princ. }}^{(n)}(\Delta)\right)$ is an operator of order $\leqslant n-1$ since the operator $\Delta \in \Delta \in \mathcal{D}_{\lambda}^{(n)}$ has the order $\leqslant n$. Thus we see that the DLO-pencil lifting defined on operators of order $\leqslant n$ can be decomposed into a sum of $n+1 o j$-equivariant maps:

$$
\widehat{\Pi}_{\lambda}^{D L O}=\widehat{\Pi}_{n, \lambda}+\widehat{\Pi}_{n-1, \lambda}+\cdots+\widehat{\Pi}_{0, \lambda}
$$

and respectively an arbitrary operator $\Delta \in \mathcal{D}_{\lambda}^{(n)}\left(\mathbb{R}^{d}\right)$ can be decomposed in the sum of the $n+1$ operators

$$
\Delta=\Delta_{n}+\Delta_{n-1}+\cdots+\Delta_{0},
$$

such that

$$
\widehat{\Pi}_{\lambda}^{\mathrm{DLO}}(\Delta)=\widehat{\Pi}_{n, \lambda}\left(\Delta_{n}\right)+\widehat{\Pi}_{n-1, \lambda}\left(\Delta_{n-1}\right)+\cdots+\widehat{\Pi}_{0, \lambda}\left(\Delta_{0}\right),
$$

where $\Delta_{n}=\left.\widehat{\Pi}_{n, \lambda}(\Delta)\right|_{\widehat{w}=\lambda}$ and inductively $\Delta_{n-k}=\left.\widehat{\Pi}_{n-k, \lambda}\left(\Delta-\Delta_{n}-\cdots-\Delta_{n-k+1}\right)\right|_{\widehat{w}=\lambda}$, and

$$
\widehat{\Pi}_{\lambda}^{\mathrm{DLO}}(\Delta)=\widehat{\Pi}_{n, \lambda}\left(\Delta_{n}\right)+\widehat{\Pi}_{n-1, \lambda}\left(\Delta_{n-1}\right)+\cdots+\widehat{\Pi}_{1, \lambda}\left(\Delta_{1}\right)+\widehat{\Pi}_{0, \lambda}\left(\Delta_{0}\right)
$$

(In fact $\left.\widehat{\Pi}_{\lambda}^{\mathrm{DLO}}\left(\Delta_{k}\right)=\widehat{\Pi}_{k, \lambda}\left(\Delta_{k}\right)\right)$.

All the operators of the pencil $\widehat{\Pi}_{\lambda}^{\mathrm{DLO}}\left(\Delta_{n}\right)$ including the operator $\Delta_{n}$ have the same full symbol which is equal to their principal symbol: If in decomposition (41), principal symbol of $\Delta_{k}$ is equal to $S^{i_{1} \ldots i_{k}} \xi_{i_{1}} \ldots \xi_{i_{k}}$ then for an arbitrary weight $\mu$

$$
\sigma_{\mu}\left(\left.\widehat{\Pi}_{n, \lambda}\left(\Delta_{n}\right)\right|_{\widehat{w}=\mu}\right)=S^{i_{1} \ldots i_{k}} \xi_{i_{1}} \ldots \xi_{i_{k}} .
$$

One can see that all operators of this pencil and their symbol are eigenvectors of the Casimir operator of the Lie algebra proj $\left(\mathbb{R}^{d}\right)$ with the same eigenvalue.

Example 5.1. Consider second order operator $\Delta=S^{i k}(x) \partial_{i} \partial_{k}+A^{i}(x) \partial_{i}+F(x) \in \mathcal{D}_{\lambda}^{(2)}\left(\mathbb{R}^{d}\right)$ acting on densities of weight $\lambda$.

The operators $\widehat{\Pi}_{2, \lambda}, \widehat{\Pi}_{1, \lambda}$ and $\widehat{\Pi}_{0, \lambda}$ on the algebra of densities (operator pencils) are defined by equation (40).

Consider the decomposition $\Delta=\Delta_{2}+\Delta_{1}+\Delta_{0}$. Due to the formulae above and equation (38) we have

$$
\begin{gathered}
\Delta_{2}=S^{i k}(x) \partial_{i} \partial_{k}+a_{d}(\lambda) \partial_{k} S^{k i}(x) \partial_{i}+b_{d}(\lambda) \partial_{k} \partial_{i} S^{k i}(x), \\
\Delta_{1}=\left(A^{i}(x)-a_{d}(\lambda) \partial_{k} S^{k i}(x)\right) \partial_{i}+\lambda\left(\partial_{i} A^{i}(x)-a_{d}(\lambda) \partial_{i} \partial_{k} S^{k i}(x)\right), \\
\Delta_{0}=F(x)-\lambda\left(\partial_{i} A^{i}(x)-a_{d}(\lambda) \partial_{i} \partial_{k} S^{k i}(x)\right)-b_{d}(\lambda) \partial_{k} \partial_{i} S^{k i}(x)
\end{gathered}
$$

and respectively

$$
\widehat{\Pi}_{\lambda}^{\mathrm{DLO}}(\Delta)=\widehat{\Delta}_{2}+\widehat{\Delta}_{1}+\widehat{\Delta}_{0}
$$

where

$$
\widehat{\Delta}_{2}=\widehat{\Pi}_{\lambda}^{\mathrm{DLO}}\left(\Delta_{2}\right)=S^{i k}(x) \partial_{i} \partial_{k}+a_{d}(\widehat{w}) \partial_{k} S^{k i} \partial_{i}+b_{d}(\widehat{w}) \partial_{k} \partial_{i} S^{k i}(x),
$$




$$
\begin{gathered}
\widehat{\Delta}_{1}=\widehat{\Pi}_{\lambda}^{\mathrm{DLO}}\left(\Delta_{1}\right)=\left(A^{i}(x)-a_{d}(\lambda) \partial_{k} S^{k i}(x)\right) \partial_{i}+\widehat{w}\left(\partial_{i} A^{i}(x)-a_{d}(\lambda) \partial_{i} \partial_{k} S^{k i}(x)\right), \\
\widehat{\Delta}_{0}=\widehat{\Pi}_{\lambda}^{\mathrm{DLO}}\left(\Delta_{0}\right)=\Delta_{0}=F(x)-\lambda \partial_{i} A^{i}(x)+\left(\lambda a_{d}(\lambda)-b_{d}(\lambda)\right) \partial_{i} \partial_{k} S^{k i}(x) .
\end{gathered}
$$

(Polynomials $a_{d}, b_{d}$ are defined by equations (39).)

Now using the decomposition above we consider the following dressing of the DLO-pencil lifting.

Pick an arbitrary $n, \lambda$ and take an arbitrary lower triangular $(n+1 \times n+1)$ matrix of numbers $\left\|a_{i j}\right\|$, where $i, j=0,1,2,3, \ldots$ such that in every row at least one of the elements is not equal to zero

$$
\left\|a_{i j}\right\|:\left\{\begin{array}{l}
a_{i j}=0, \text { if } j>i \\
\text { in every row there exists a non-zero element }
\end{array} .\right.
$$

Now take the DLO-pencil lifting of the space $\mathcal{D}_{\lambda}^{(n)}\left(\mathbb{R}^{d}\right)$ and assign to every matrix obeying the conditions above the pencil: $\widehat{\Pi}_{\lambda}^{\left\|a_{i j}\right\|}$ such that

$$
\begin{gathered}
\widehat{\Pi}_{\lambda}^{\left\|a_{i j}\right\|}(\Delta)=\sum_{i=0}^{n} \frac{P_{i}(\widehat{w})}{P_{i}(\lambda)} \widehat{\Pi}_{\lambda}^{\mathrm{DLO}}\left(\Delta_{n-i}\right)= \\
\widehat{\Pi}^{\mathrm{DLO}}\left(\Delta_{n}\right)+\frac{a_{10}+a_{11} \widehat{w}}{a_{10}+a_{11} \lambda} \widehat{\Pi}_{\lambda}^{\mathrm{DLO}}\left(\Delta_{n-1}\right)+\frac{a_{20}+a_{21} \widehat{w}+a_{22} \widehat{w}^{2}}{a_{20}+a_{21} \lambda+a_{22} \lambda^{2}} \widehat{\Pi}_{\lambda}^{\mathrm{DLO}}\left(\Delta_{n-2}\right)+\ldots,
\end{gathered}
$$

where $\Delta=\Delta_{n}+\Delta_{n-1}+\cdots+\Delta_{1}+\Delta_{0}$ is decomposition (41) and the polynomials $P_{i}(\lambda)$ are defined by the matrix $\left\|a_{i j}\right\|: P_{i}(\lambda)=\sum a_{i j} \lambda^{j}$.

One can see that all these pencil liftings are regular pencil liftings. If for two matrices $\left\|a_{i k}\right\|$ and $\left\|a_{i k}^{\prime}\right\|$ obeying conditions (44) all rows are proportional then the corresponding pencils coincide. This formula describes the family of pencil liftings parameterised by

$$
\mathbb{R} P^{1} \times \mathbb{R} P^{2} \times \ldots \mathbb{R} P^{n}
$$

On the other hand let $\widehat{\Pi}_{\lambda}$ be an arbitrary regular proj $\left(\mathbb{R}^{d}\right)$-equivariant pencil lifting on the space $\mathcal{D}_{\lambda}^{(n)}(M)$. It follows from decomposition (41) that for every $k,(k=1, \ldots, n$, $\left.\Delta=\Delta_{n}+\Delta_{n-1}+\cdots+\Delta_{0}\right),\left.\widehat{\Pi}_{\lambda}\left(\Delta_{k}\right)\right|_{\widehat{w}=\mu}=F_{n-k}(\mu) \Pi_{k}\left(\Delta_{k}\right)$, where $F_{n-k}$ is a polinomial such $F_{n-k}(\lambda)=1$. The conditions of regularity of the pencil dictate that $F_{n-k}$ are polynomials of order $\leqslant n-k, F_{n-k}(\widehat{w})=\sum_{r=0}^{k} a_{n r} \widehat{w}^{r}$. Thus we come to the fact that $\widehat{\Pi}_{\lambda}=\widehat{\Pi}^{\left\|a_{i k}\right\|}$. We come to the proposition

Proposition 3. For arbitrary n, $\lambda$ equation (44) describes the family of regular proj-equivariant pencil liftings of operators $\mathcal{D}_{\lambda}^{(n)}(M)$. These are all regular proj-equivariant pencil liftings of $\mathcal{D}_{\lambda}^{(n)}(M)$.

One can see from decomposition (41) and uniqueness arguments that the DLO-pencil lifting is self-adjoint on operators $\Delta_{2 k}$ and anti-self-adjoint on operators $\Delta_{2 k+1}$. On the other hand the polynomial $P(\widehat{w})$, a vertical operator, is self-adjoint iff $P_{k}(\widehat{w})$ is an even polynomial of the variable $\widehat{w}^{\prime}=\widehat{w}-\frac{1}{2}$ and it is anti-self-adjoint if it is an odd polynomial of $\widehat{w}^{\prime}=\widehat{w}-\frac{1}{2}$ since $\widehat{w}^{\prime}=\widehat{w}-\frac{1}{2}$ is an anti-self-adjoint operator. Thus we come to a description of self-adjoint and anti-self-adjoint projective liftings. 
Proposition 4. Let $\widehat{\Pi}$ be a regular proj-equivariant lifting on the space $\mathcal{D}_{\lambda}^{(n)}(M)$. Then this lifting is self adjoint, i.e. it takes values in self-adjoint operator pencils, if $n$ is even and in the equation (44) for all the polynomials $\left\{P_{i}(\widehat{w})\right\}$

$$
P_{i}(\widehat{w})^{*}=(-1)^{i} P_{i}(\widehat{w})
$$

i.e. polynomials $P_{2 i}$ are self-adjoint

$$
P_{2 i}(\widehat{w})=\sum c_{k}\left(\widehat{w}-\frac{1}{2}\right)^{2 k}
$$

and polynomials $P_{2 i+1}$ are anti-self-adjoint

$$
P_{2 i+1}(\widehat{w})=\sum c_{k}\left(\widehat{w}-\frac{1}{2}\right)^{2 k+1} .
$$

Respectively the regular proj-equivariant pencil lifting is anti-self-adjoint if $n$ is odd and if all the conditions above for polynomials are fullfilled.

It is very illuminating to consider the following example of self-adjoint regular proj-liftings for second order operators.

An anti-self-adjoint polynomial of order $\leqslant 1$ is proportional to $2 \widehat{w}-1$ and a self-adjoint polynomial of order $\leqslant 2$ is proportional to

$$
a+b\left(\widehat{w}-\frac{1}{2}\right)^{2}=a+\frac{b}{4}+b \widehat{w}(\widehat{w}-1) .
$$

Hence an arbitrary self-adjoint regular proj-equivariant pencil lifting of second order operators on densities of weight $\lambda$ is of the form

$$
\widehat{\Pi}_{\lambda}^{\mathrm{DLO}}\left(\Delta_{2}\right)+\frac{2 \widehat{w}-1}{2 \lambda-1} \widehat{\Pi}_{\lambda}^{\mathrm{DLO}}\left(\Delta_{1}\right)+\frac{p+q \widehat{w}(\widehat{w}-1)}{p+q \lambda(\lambda-1)} \Delta_{0},
$$

where $\Delta=\Delta_{2}+\Delta_{1}+\Delta_{0}$ is decomposition (411). We come to a projective line of self-adjoint liftings on densities of weight $\lambda(p \neq 0$ or $q \neq 0)$.

Take an arbitrary second order operator acting on densities of weight $\lambda$

$$
\Delta=S^{i k}(x) \partial_{i} \partial_{k}+A^{i}(x) \partial_{i}+F(x) .
$$

According to equations (42) in example 5.1 we have

$$
\begin{gathered}
\widehat{\Pi}^{([p: q])}(\Delta)=(\underbrace{S^{i k}(x) \partial_{i} \partial_{k}+a_{d}(\widehat{w}) \partial_{k} S^{k i}(x) \partial_{i}+b_{d}(\widehat{w}) \partial_{i} \partial_{k} S^{k i}(x)}_{\widehat{\Delta}_{2}=\widehat{\Pi}_{\lambda}^{\mathrm{DL}}\left(\Delta_{2}\right)})+ \\
+\frac{2 \widehat{w}-1}{2 \lambda-1}(\underbrace{\left(A^{i}(x)-a_{d}(\lambda) \partial_{k} S^{k i}(x)\right) \partial_{i}+\widehat{w}\left(\partial_{i} A^{i}(x)-a_{d}(\lambda) \partial_{i} \partial_{k} S^{k i}(x)\right)}_{\widehat{\Delta}_{1}=\widehat{\Pi}_{\lambda}^{D L O}\left(\Delta_{1}\right)})+ \\
+\frac{p+q \widehat{w}(\widehat{w}-1)}{p+q \lambda(\lambda-1)}(\underbrace{F(x)-\lambda \partial_{i} A^{i}(x)+\left(\lambda a_{d}(\lambda)-b_{d}(\lambda)\right) \partial_{k} \partial_{i} S^{k i}(x)}_{\Delta_{0}}) .
\end{gathered}
$$


We now compare pencils in this family with the canonical self-adjoint lifting given by theorem 1. Recall that for the operator $\Delta=S^{i k}(x) \partial_{i}+A^{i}(x) \partial_{i}+F(x)$ the self-adjoint canonical lifting is equal to

$$
\widehat{\Delta}=S^{i k}(x)+\partial_{k} S^{i k}(x) \partial_{i}+(2 \widehat{w}-1) B^{i}(x) \partial_{i}+\widehat{w} \partial_{i} B^{i}(x)+\widehat{w}(\widehat{w}-1) C(x),
$$

where

$$
B^{i}(x)=\frac{A^{i}(x)-\partial_{k} S^{i k}(x)}{2 \lambda-1}, C(x)=\frac{F(x)}{\lambda(\lambda-1)}-\frac{\partial_{i} A^{i}(x)-\partial_{i} \partial_{k} S^{k i}(x)}{(\lambda-1)(2 \lambda-1)} .
$$

(See equations (17) and (24). We suppose that $\lambda \neq 0, \frac{1}{2}, 1$.)

Note that in the case when parameter for lifting (47) is equal to $[p: q]=[0: 1]$. In this case the lifted operator $\widehat{\Delta}=\widehat{\Pi}^{([0: 1])}(\Delta)$ obeys the normalisation condition $\widehat{\Delta}(1)=0$, hence due to theorem 1 it is equal to self-adjoint lifting (48).

Now comparing an arbitrary self-adjoint operator $\widehat{\Pi}^{([p: q])}(\Delta)$ from the family (47) with the canonical self-adjoint operator $\widehat{\Delta}=\widehat{\Pi}^{([0: 1])}$ we see that their difference is a vertical operator. Hence we come to decomposition:

$$
\widehat{\Pi}^{([p: q])}(\Delta)=\widehat{\Delta}+\left(\frac{p+q \widehat{w}(\widehat{w}-1)}{p+q \lambda(\lambda-1)}-\frac{\widehat{w}(\widehat{w}-1)}{\lambda(\lambda-1)}\right)(\underbrace{F(x)-\lambda \partial_{i} A^{i}(x)+\left(\lambda a_{d}(\lambda)-b_{d}(\lambda)\right) \partial_{i} \partial_{k} S^{k i}(x)}_{\delta}) .
$$

The expression

$$
\mathcal{S}=\mathcal{S}_{\Delta}=F(x)-\lambda \partial_{i} A^{i}(x)+\left(\lambda a_{d}(\lambda)-b_{d}(\lambda)\right) \partial_{i} \partial_{k} S^{k i}(x),
$$

due to its construction transforms as a scalar under arbitrary projective transformations. From the uniqueness of the canonical self-adjoint pencil it follows that under arbitrary diffeomorphisms $\mathcal{S}$ transforms non-trivially. We come to a cocycle which is a multidimensional analogue of the usual Schwarzian (see the book [12]).

Remark 9. It is instructive to compare calculations in this example with those in $\S 7.1$ of the book [12].

Let us finish with a remark. If a pencil lifting factors through the space of symbols then it is induced by a full symbol calculus (see the Introduction). In this article the only such pencil lifting is the DLO-lifting. The projective quantisation map inducing this lifting is unique. However there are many regular proj-equivariant liftings of differential operators, these are maps between non-commutative algebras.

\section{REFERENCES}

[1] A.Biggs "Geometric structures on the algebra of densities" PhD Thesis, The University of Manchester, 2014 .

[2] A.Biggs, H.M.Khudaverdian "Operator pencil passing through a given operator," J. Math. Phys. 54, 123503, (2013)

[3] Cristian Duval, Pierre Lecomte, Valentin Ovsienko, "Conformally equivariant quantisation: existence and uniqueness," Ann.Inst.Fourier 49, 1999 (1999).

[4] C.Duval, V. Yu. Ovsienko, "Space of second order linear differential operators as a module over the Lie algebra of vector fields," Advances in Mathematics 132, 316 (1997).

[5] H.M.Khudaverdian "Kaluza-Klein theory revisited: projective structures and differential operators on algebra of densities" math-arXiv 1312.5208 
[6] H.M.Khudaverdian, T.Voronov "On Odd Laplace operators II," In Amer.Math.Soc.Transl.(2), 212, 179 (2004).

[7] H.M.Khudaverdian, T.Voronov "Geometry of differential operators of second order, the algebra of densities, and groupoids," J. Geom. Phys. 64, 31 (2013). (See also preprint of Max-Planck-Institut for Math., 73, (2011).)

[8] H.M.Khudaverdian, T.Voronov "Geometric constructions on algebra of densities" matharXiv:1310.0784.

[9] P.Lecomte, P.Mathonet and E.Tousset, "Comparison of some modules of the Lie algebra of vector fields," Indag.Mathem., N.S. 7, 461 (1996).

[10] P.Lecomte, V.Yu.Ovsienko, "Projetive equivariant symbol calculus," Lett.Math.Phys. 49, 173 (1999).

[11] P.Mathonet, "Interwinning operators between some spaces of differential operators on a manifold," Communications in Algebra 27, 755 (1999).

[12] V. Ovsienko, S.Tabachnikov Projective Differential Geometry Old and New From Schwarzian Derivative to the Cohomology of Diffeomorphism Groups. (Cambridge University Press, 2005).

[13] J.Peetre "Une caractérisation abstraite des opérateurs différentiels," Math.Scand. 7, 211(1959), 7 and 8, 116 (1960).

School of Mathematics, University of Manchester, Oxford Road, Manchester M13 9PL, $\mathrm{UK}$

E-mail address: khudian@manchester.ac.uk, adam.biggs@student.manchester.ac.uk 